\title{
Boundary-layer stability of supercritical fluids in the vicinity of the Widom line
}

\author{
Jie Ren ${ }^{1} \uparrow$, Olaf Marxen ${ }^{2}$ and Rene Pecnik ${ }^{1, \dagger}$ \\ ${ }^{1}$ Process and Energy Department, Delft University of Technology, Leeghwaterstraat 39, \\ 2628 CB Delft, The Netherlands \\ ${ }^{2}$ Department of Mechanical Engineering Sciences, University of Surrey, Guildford GU2 7XH, UK
}

(Received 30 November 2018; revised 21 March 2019; accepted 24 April 2019; first published online 28 May 2019)

We investigate the hydrodynamic stability of compressible boundary layers over adiabatic walls with fluids at supercritical pressure in the proximity of the Widom line (also known as the pseudo-critical line). Depending on the free-stream temperature and the Eckert number that determines the viscous heating, the boundary-layer temperature profile can be either sub-, trans- or supercritical with respect to the pseudo-critical temperature, $T_{p c}$. When transitioning from sub- to supercritical temperatures, a seemingly continuous phase change from a compressible liquid to a dense vapour occurs, accompanied by highly non-ideal changes in thermophysical properties. Using linear stability theory (LST) and direct numerical simulations (DNS), several key features are observed. In the sub- and supercritical temperature regimes, the boundary layer is substantially stabilized the closer the free-stream temperature is to $T_{p c}$ and the higher the Eckert number. In the transcritical case, when the temperature profile crosses $T_{p c}$, the flow is significantly destabilized and a co-existence of dual unstable modes (Mode II in addition to Mode I) is found. For high Eckert numbers, the growth rate of Mode II is one order of magnitude larger than Mode I. An inviscid analysis shows that the newly observed Mode II cannot be attributed to Mack's second mode (trapped acoustic waves), which is characteristic in high-speed boundary-layer flows with ideal gases. Furthermore, the generalized Rayleigh criterion (also applicable for non-ideal gases) unveils that, in contrast to the trans- and supercritical regimes, the subcritical regime does not contain an inviscid instability mechanism.

Key words: boundary layer stability, compressible boundary layers

\section{Introduction}

Complex molecular interactions close to the vapour-liquid critical point of a substance are responsible for the highly non-ideal thermodynamic behaviour. In this thermodynamic region, fluids exhibit significant deviations from ideal-gas law behaviour and their properties can be utilized to increase productivity and efficiency of

$\dagger$ Email addresses for correspondence: j.ren-1@tudelft.nl, renjies950@gmail.com, r.pecnik@tudelft.nl 
many technical processes. For instance, significant improvements of turbine efficiency can be achieved by using complex molecular fluids in organic Rankine cycles (Brown \& Argrow 2000); injecting fuels at supercritical conditions can be employed to obtain higher efficiency of mixing and combustion in air breathing and liquid rocket engines (Wang \& Yang 2017); and power cycles operating with supercritical carbon dioxide offer the potential to drastically increase thermal efficiency to enable competitive utility scale renewable electricity production. Despite this fundamental importance both in science and industrial applications, flow instability and laminar-to-turbulent transition with fluids close to their vapour-liquid critical point still remain unexplored.

A large body of literature has been concerned with understanding and controlling transition with idealized fluids, such as incompressible and ideal-gas compressible shear flows. While compressibility significantly enriches the physics of flow transition (Fedorov 2011; Zhong \& Wang 2012), the interest in vehicles travelling at supersonic and hypersonic speeds has sparked research to unveil complex phenomena (high-temperature effects, shock waves, entropy layers, etc.) that influence the excitation and growth of instabilities and consequently transition to turbulence.

Early work on instability of ideal-gas compressible boundary layers started in the 1940s. In the same way as the Rayleigh criterion for incompressible flows states that the necessary condition for instability is the presence of an inflection in the velocity profile, Lees \& Lin (1946) showed that in compressible flows the generalized inflection point is a necessary condition of an inviscid instability to occur. Mack (1984) showed that compressible boundary layers at high speeds reveal that there are higher modes that can be present in the boundary layer, which belong to the family of trapped acoustic waves. For the case of a laminar insulated boundary layer, it turns out that the first higher mode (Mack's second mode) exceeds the growth rate of the first viscous mode around a free-stream Mach number of 4 .

High-temperature chemical effects on boundary-layer stability and transition have been investigated since the 1990s, addressing the needs imposed by the re-entry of hypersonic vehicles. These effects, often referred to as real-gas effects, include vibrational excitation, dissociation and recombination of gas species, ionization, radiation and surface ablation. The instability of a Mach 10 boundary layer was introduced and discussed by Malik \& Anderson (1991). Based on linear stability analysis, these effects are shown to stabilize the first mode and destabilize Mack's second mode. Subsequent studies had been extended to account for chemical and/or thermal non-equilibrium effects (Stuckert \& Reed 1994; Hudson, Chokani \& Candler 1997), different chemical reaction models (Lyttle \& Reed 2005; Franko, MacCormack \& Lele 2010), surface ablation effects (Mortensen \& Zhong 2016) and coupling with roughness induced transition (Stemmer, Birrer \& Adams 2017). Apart from earlier linear modal stability theory, parabolized stability equations (PSE) (Chang et al. 1997; Johnson \& Candler 1999; Malik 2003) as well as direct numerical simulations (DNS) (Marxen et al. 2013; Marxen, Iaccarino \& Magin 2014; Wang 2017) have been developed and applied to understand the related transition mechanisms.

Apart from the high-temperature chemical effects, stratifications in thermodynamic or/and transport properties can substantially influence the stability (see review by Govindarajan \& Sahu (2014), and references therein). These stratifications exist both naturally (e.g. in the Earth's outer core) and artificially (e.g. exert wall heating/cooling), revealing some of the non-ideal-gas effects. The linear instabilities in parallel shear flows have received most of the attention so far. For example, plane Poiseuille flows can be markedly stabilized by decreasing wall viscosity (Sameen \& Govindarajan 2007). Plane Couette flows are known to be stable to modal instabilities 
at any Reynolds number. It has been recently confirmed that a vertical density stratification can drive the flow modally unstable at a moderate Reynolds number (Facchini et al. 2018).

When a fluid is operating near its thermodynamic critical point, strong property stratifications occur that must be modelled using complex equations of state. Supercritical fluid flows have received growing interests in various industrial applications (Brunner 2010) and recent studies have focused on characterizing turbulence and heat transfer. For example, Pecnik \& Patel (2017) derived an alternative formulation of the turbulent kinetic energy equation using semi-local quantities. They showed that the semi-local scaling, as proposed by Huang, Coleman \& Bradshaw (1995), can also be applied to conservation laws, such as the turbulent kinetic energy equation. Using this approach, it is then possible to quantify turbulence modulation related to density and viscosity stratifications using the semi-local Reynolds number. Kawai (2016) performed the first DNS on supercritical turbulent boundary-layer flow with transcritical temperature, and showed that the turbulent mass flux terms in the turbulent kinetic energy equation largely exceed values as observed for ideal gas at the same free-stream Mach numbers. In terms of heat transfer, as concluded in a recent review (Pizzarelli 2018), future applications are still limited by the poor understanding and prediction of heat transfer deterioration in supercritical fluids. The linear stability of flows with such highly non-ideal fluids has only been considered recently by Ren, Fu \& Pecnik (2019) for plane Poiseuille flows. It was found that compared to ideal gases at the same conditions, the non-ideal gas can become more stable/unstable, or even inviscid unstable in different thermodynamic regimes.

This study aims to investigate the stability of boundary-layer flows with fluids close to the critical point, through linear stability theory (LST), DNS and inviscid analysis. To account for the full non-ideal-gas effects, one must take the non-ideal equation of state into consideration as well as the complicated functions of thermodynamic/transport properties in terms of the thermodynamic state, which can be determined by two independent thermodynamic quantities (temperature, density, pressure etc.). We study boundary-layer flows with carbon dioxide $\left(\mathrm{CO}_{2}\right)$ at a constant pressure of $80 \mathrm{bar}$, which is above the critical pressure (73.9 bar). The flow conditions are such chosen that different thermodynamic regimes of interests shall be well revealed. In $\S 2$, the formulation of the base flow and stability analysis as well as the related numerical methods are introduced. The cases investigated and discussions on the base flow are provided in $\S 3$, followed by the linear stability analysis, direct numerical simulation and inviscid analysis in $\S 4$. The study is concluded in $\S 5$.

\section{Formulation and numerical details}

\subsection{Flow conservation equations}

The laws of conservation of mass, momentum and energy (known as the NavierStokes equations), in differential and dimensionless form, are

$$
\left.\begin{array}{c}
\frac{\partial \rho}{\partial t}+\frac{\partial\left(\rho u_{j}\right)}{\partial x_{j}}=0, \\
\frac{\partial\left(\rho u_{i}\right)}{\partial t}+\frac{\partial\left(\rho u_{i} u_{j}+p \delta_{i j}-\tau_{i j}\right)}{\partial x_{j}}=0, \\
\frac{(\rho E)}{\partial t}+\frac{\partial\left(\rho E u_{j}+p u_{j}+q_{j}-u_{i} \tau_{i j}\right)}{\partial x_{j}}=0,
\end{array}\right\}
$$


where $x_{i}=(x, y, z)$ are the coordinates in the streamwise, wall-normal and spanwise directions, $u_{i}=(u, v, w)$ are the corresponding velocity components, $t$ the time, $\rho$ the fluid density, $E=e+u_{i} u_{i} / 2$ the total energy, $e$ the internal energy and $p$ is the pressure. The viscous stress tensor, $\tau_{i j}$, and the heat flux vector, $q_{j}$, are given by

$$
\left.\begin{array}{c}
\tau_{i j}=\frac{\mu}{R e_{\infty}}\left(\frac{\partial u_{i}}{\partial x_{j}}+\frac{\partial u_{j}}{\partial x_{i}}\right)+\frac{\lambda}{R e_{\infty}} \delta_{i j} \frac{\partial u_{k}}{\partial x_{k}}, \\
q_{j}=-\frac{\kappa}{R e_{\infty} P r_{\infty} E c_{\infty}} \frac{\partial T}{\partial x_{j}} .
\end{array}\right\}
$$

Here $\mu$ is the dynamic viscosity, $\lambda=\mu_{b}-2 / 3 \mu$ the second viscosity, $\mu_{b}$ the bulk viscosity and $\kappa$ is the thermal conductivity. It is known that $\mu_{b}$ has a very limited effect on the linear stability of channel flows (Ren et al. 2019). Results presented in the following sections are subjected to $\mu_{b}=0$. An additional assumption is that buoyancy effects are not considered. The equations above have been non-dimensionalized by reference values, as follows

$$
\left.\begin{array}{c}
u=\frac{u^{*}}{u_{\infty}^{*}}, \quad x_{i}=\frac{x_{i}^{*}}{l_{0}^{*}}, \quad t=\frac{t^{*} u_{\infty}^{*}}{l_{0}^{*}}, \quad p=\frac{p^{*}}{\rho_{\infty}^{*} u_{\infty}^{* 2}}, \quad \rho=\frac{\rho^{*}}{\rho_{\infty}^{*}}, \\
T=\frac{T^{*}}{T_{\infty}^{*}}, \quad E=\frac{E^{*}}{u_{\infty}^{* 2}}, \quad \mu=\frac{\mu^{*}}{\mu_{\infty}^{*}}, \quad \kappa=\frac{\kappa^{*}}{\kappa_{\infty}^{*}}, \quad
\end{array}\right\}
$$

which leads to the definition of the Reynolds number, $R e_{\infty}$, Prandtl number, $P r_{\infty}$, Eckert number, $E c_{\infty}$, and the Mach number, $M a_{\infty}$ (all based on free-stream parameters)

$$
R e_{\infty}=\frac{\rho_{\infty}^{*} u_{\infty}^{*} l_{0}^{*}}{\mu_{\infty}^{*}}, \quad P r_{\infty}=\frac{\mu_{\infty}^{*} C_{p \infty}^{*}}{\kappa_{\infty}^{*}}, \quad E c_{\infty}=\frac{u_{\infty}^{* 2}}{C_{p \infty}^{*} T_{\infty}^{*}}, \quad M a_{\infty}=\frac{u_{\infty}^{*}}{a_{\infty}^{*}} .
$$

The subscript $\infty$ denotes free-stream values, superscript $*$ stands for dimensional variables, $l_{0}^{*}$ is a chosen length scale, $a_{\infty}^{*}$ is the speed of sound in the free stream. Note that for an ideal gas $E c_{\infty}=(\gamma-1) M a_{\infty}^{2}$, where $\gamma$ is the heat capacity ratio. In linear stability theory, $l_{0}^{*}$ is chosen to be the local boundary-layer thickness scale $\delta^{*}$, which results in the definition of $R_{\delta}$,

$$
\delta^{*}=\left(\frac{\mu_{\infty}^{*} x^{*}}{\rho_{\infty}^{*} u_{\infty}^{*}}\right)^{1 / 2}, \quad R e_{\delta}=\frac{\rho_{\infty}^{*} u_{\infty}^{*} \delta^{*}}{\mu_{\infty}^{*}}=\left(\frac{\rho_{\infty}^{*} u_{\infty}^{*} x^{*}}{\mu_{\infty}^{*}}\right)^{1 / 2} .
$$

Here, $\delta^{*}$ measures the order of boundary-layer thickness over a flat plate. For example, applying the Blasius solution, the displacement thickness $\delta_{1}^{*} \approx 1.721 \delta^{*}$, momentum thickness $\delta_{2}^{*} \approx 0.664 \delta^{*}$ (Schlichting \& Gersten 2017).

\subsection{The laminar base flow}

The self-similar solution to the boundary-layer equation over a flat plate is employed in this work. It serves as the base flow for the stability analysis as well as for the initial state of the DNS. Applying the boundary-layer assumption and a routine 
order-of-magnitude analysis of the dimensional Navier-Stokes $(\mathrm{N}-\mathrm{S})$ equations, the boundary-layer equations read,

$$
\left.\begin{array}{c}
\frac{\partial\left(\rho^{*} u^{*}\right)}{\partial x^{*}}+\frac{\partial\left(\rho^{*} v^{*}\right)}{\partial y^{*}}=0, \\
\rho^{*} u^{*} \frac{\partial u^{*}}{\partial x^{*}}+\rho^{*} v^{*} \frac{\partial u^{*}}{\partial y^{*}}+\frac{\mathrm{d} p_{\infty}^{*}}{\mathrm{~d} x^{*}}-\frac{\partial}{\partial y^{*}}\left(\mu^{*} \frac{\partial u^{*}}{\partial y^{*}}\right)=0, \\
\rho^{*} u^{*} \frac{\partial h^{*}}{\partial x^{*}}+\rho^{*} v^{*} \frac{\partial h^{*}}{\partial y^{*}}-u^{*} \frac{\mathrm{d} p_{\infty}^{*}}{\mathrm{~d} x^{*}}-\frac{\partial}{\partial y^{*}}\left(\kappa^{*} \frac{\partial T^{*}}{\partial y^{*}}\right)-\mu^{*}\left(\frac{\partial u^{*}}{\partial y^{*}}\right)^{2}=0 .
\end{array}\right\}
$$

Introducing the Lees-Dorodnitsyn transformation (see introduction in Anderson $\mathrm{Jr}$ 2000; Schlichting \& Gersten 2017)

$$
\left.\begin{array}{c}
\mathrm{d} \xi=\rho_{\infty}^{*} \mu_{\infty}^{*} u_{\infty}^{*} \mathrm{~d} x^{*}, \\
\mathrm{~d} \eta=\frac{\rho^{*} u_{\infty}^{*}}{\sqrt{2 \xi}} \mathrm{d} y^{*},
\end{array}\right\}
$$

for the boundary-layer equations, yields the transformed ordinary differential equations (ODE) for $f$ and $g$ given as,

$$
\left.\begin{array}{c}
\frac{\mathrm{d}}{\mathrm{d} \eta}\left(C_{l} \frac{\mathrm{d}^{2} f}{\mathrm{~d} \eta^{2}}\right)+f \frac{\mathrm{d}^{2} f}{\mathrm{~d} \eta^{2}}=0, \\
\frac{\mathrm{d}}{\mathrm{d} \eta}\left(\frac{C_{l}}{P r_{l}} \frac{\mathrm{d} g}{\mathrm{~d} \eta}\right)+f \frac{\mathrm{d} g}{\mathrm{~d} \eta}+C_{l} \frac{u_{\infty}^{* 2}}{h_{t \infty}^{*}}\left(\frac{\mathrm{d}^{2} f}{\mathrm{~d} \eta^{2}}\right)^{2}=0,
\end{array}\right\}
$$

where

$$
\frac{\mathrm{d} f}{\mathrm{~d} \eta}=\frac{u^{*}}{u_{\infty}^{*}}, \quad g=\frac{h_{t}^{*}}{h_{t \infty}^{*}}, \quad C_{l}=\frac{\rho^{*} \mu^{*}}{\rho_{\infty}^{*} \mu_{\infty}^{*}}, \quad P r_{l}=\frac{\mu^{*} C_{p}^{*}}{\kappa^{*}} .
$$

Here, $f$ and $g$ are unary functions of the transformed coordinate $\eta$, while $h_{t}$ denotes the total enthalpy. The above ODEs are numerically integrated using the fourth-order Runge-Kutta scheme subjected to adiabatic wall boundary conditions. During the integration process, a one-dimensional (1-D) look-up table (see appendix A) is used to calculate $C_{l}$ and $P r_{l}$ from the static temperature that can be obtained from the total enthalpy $g$.

\subsection{Linear stability theory}

The linear stability equations for the non-ideal gas have been derived and documented in Ren et al. (2019). It is known that for simple compressible systems (e.g. single phase, pure substances and uniform mixtures of non-reacting gases), the thermodynamic state is defined by two independent thermodynamic properties. We choose $\rho$ and $T$ as the two independent thermodynamic quantities, while the remaining thermodynamic and transport properties (e.g. $E, p, \mu, \kappa)$ are determined as functions of $\rho$ and $T$. For example, the viscosity perturbation is given by the two-dimensional Taylor expansion,

$$
\begin{aligned}
\mu^{\prime}= & \left.\frac{\partial \mu_{0}}{\partial \rho_{0}}\right|_{T} \rho^{\prime}+\left.\frac{\partial \mu_{0}}{\partial T_{0}}\right|_{\rho} T^{\prime}+\frac{1}{2}\left(\left.\frac{\partial^{2} \mu_{0}}{\partial \rho_{0}^{2}}\right|_{T} \rho^{\prime} \rho^{\prime}+\left.\left.2 \frac{\partial}{\partial T_{0}}\right|_{\rho} \frac{\partial \mu_{0}}{\partial \rho_{0}}\right|_{T} \rho^{\prime} T^{\prime}+\left.\frac{\partial^{2} \mu_{0}}{\partial T_{0}^{2}}\right|_{\rho} T^{\prime} T^{\prime}\right) \\
& +\cdots
\end{aligned}
$$


It can be seen that in non-ideal-gas flows, the viscosity perturbation is dependent on $\partial \mu_{0} / \partial \rho_{0}$ (at constant $T$ ), which is not accounted for in the conventional empirical viscosity laws of an ideal gas (e.g. Sutherland's law and power law). The full stability equation is derived by introducing small perturbations into the $\mathrm{N}-\mathrm{S}$ equations (2.1) and subtracting the governing equations of the base flow. With the nonlinear terms neglected, the linear stability equations are formulated as

$$
\begin{aligned}
& \boldsymbol{L}_{\boldsymbol{t}} \frac{\partial \boldsymbol{q}}{\partial t}+\boldsymbol{L}_{\boldsymbol{x}} \frac{\partial \boldsymbol{q}}{\partial x}+\boldsymbol{L}_{\boldsymbol{y}} \frac{\partial \boldsymbol{q}}{\partial y}+\boldsymbol{L}_{\boldsymbol{z}} \frac{\partial \boldsymbol{q}}{\partial z}+\boldsymbol{L}_{\boldsymbol{q}} \boldsymbol{q} \\
& \quad+\boldsymbol{V}_{\boldsymbol{x} x} \frac{\partial^{2} \boldsymbol{q}}{\partial x^{2}}+\boldsymbol{V}_{\boldsymbol{x} \boldsymbol{y}} \frac{\partial^{2} \boldsymbol{q}}{\partial x \partial y}+\boldsymbol{V}_{\boldsymbol{x} z} \frac{\partial^{2} \boldsymbol{q}}{\partial x \partial z}+\boldsymbol{V}_{y \boldsymbol{y}} \frac{\partial^{2} \boldsymbol{q}}{\partial y^{2}}+\boldsymbol{V}_{y z} \frac{\partial^{2} \boldsymbol{q}}{\partial y \partial z}+\boldsymbol{V}_{z z} \frac{\partial^{2} \boldsymbol{q}}{\partial z^{2}}=0 .
\end{aligned}
$$

Here $\boldsymbol{q}=\left(\rho^{\prime}, u^{\prime}, v^{\prime}, w^{\prime}, T^{\prime}\right)^{\mathrm{T}}$ is the perturbation vector and the detailed expressions for the matrices $L_{t}, L_{x}, L_{y}, L_{z}, L_{q}, V_{x x}, V_{y y}, V_{z z}, V_{x y}, V_{y z}$ and $V_{x z}$ are functions of the dimensionless parameter (2.4), the base flow and thermodynamic and transport properties, and their detailed expressions can be found in Ren et al. (2019). The perturbation is assumed to have the normal-mode form,

$$
\boldsymbol{q}(x, y, z, t)=\hat{\boldsymbol{q}}(y) \exp (\mathrm{i} \alpha x+\mathrm{i} \beta z-\mathrm{i} \omega t)+\text { c.c. }
$$

where c.c. stands for the complex conjugate. Substituting (2.12) into (2.11) results in an eigenvalue problem. For boundary layers, we consider the spatial problem, where $\omega$ and $\beta$ are prescribed frequency and spanwise wavenumber. The real and imaginary parts of the eigenvalue $\alpha$ give the streamwise wavenumber and its local growth rate, respectively.

To solve the eigenvalue problem, Chebyshev collocation points and Chebyshev differentiation matrices are introduced to discretize the equations. The perturbations are subjected to the following boundary conditions: $u^{\prime}=v^{\prime}=w^{\prime}=0$ and $\partial T^{\prime} / \partial y=0$ at the wall $(y=0)$, while in the free stream at $y=y_{\max }, u^{\prime}=v^{\prime}=w^{\prime}=0$ and $T^{\prime}=0$.

\subsection{Direct numerical simulation}

In the context of direct numerical simulations, the $\mathrm{N}-\mathrm{S}$ equations (2.1) are numerically integrated. Wall blowing and suction are introduced to excite the Tollmien-Schlichting (T-S) waves, which are the normal-mode solutions of the linearized N-S equations (Schmid \& Henningson 2001). The amplitude of the forcing has been properly chosen (between $10^{-5}$ and $10^{-3}$ of the free-stream Mach number) such that the excited perturbations stay in the linear regime. At the wall, no-slip and adiabatic boundary conditions are applied. At the inflow the self-similar solution obtained in $\S 2.2$ is prescribed. Close to the outflow and the free-stream boundaries, a sponge region forces the solution towards the corresponding laminar state. The algorithm is based on a sixth-order compact finite-difference method with a staggered arrangement of flow variables, while the time stepping scheme is based on an explicit third-order Runge-Kutta time method. The DNS has been performed for 2-D perturbations in this study, a $N_{x} \times N_{y}=1201 \times 201$ mesh has been used for most cases and is sufficient to give a grid-independent result. The non-ideal fluid properties are incorporated using 2-D look-up tables (see appendix A). Readers may refer to Nagarajan, Lele \& Ferziger (2003) and Marxen et al. $(2011,2013)$ for numerical details.

To analyse the results from DNS and to compare them with LST, the results are Fourier transformed in time with a fundamental angular frequency of $\Omega=\omega / 2$,

$$
\hat{q}_{k}(x, y, z)=\frac{1}{N} \sum_{l=1}^{N} q\left(x, y, z, t_{l}\right) \exp \left(2 \mathrm{i} k \Omega t_{l}\right) .
$$




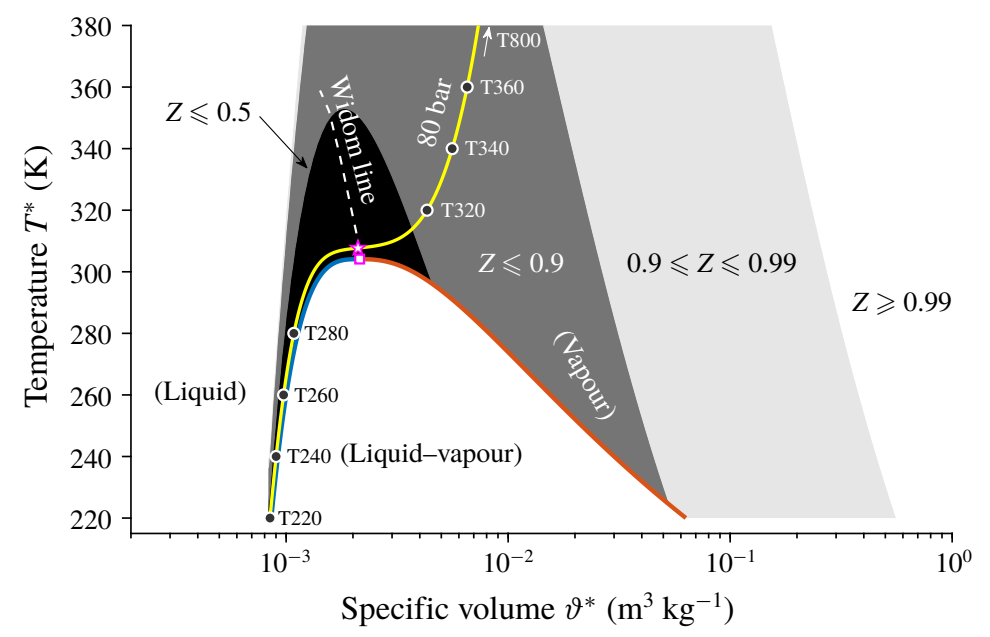

FIGURE 1. (Colour online) $T-\vartheta$ diagram of $\mathrm{CO}_{2}$ together with the critical point (magenta square), pseudo-critical point (magenta pentagram), Widom line (white dashed line), two saturation curves (blue and red thick lines) and an isobar of 80 (yellow line). The shaded area shows the contour of compressibility factor $Z$, indicating the degree of the non-ideality. The free-stream temperatures $(\mathrm{T} 220, \mathrm{~T} 240, \ldots)$ to be investigated are highlighted with circles on the isobar.

We take $N=50$ samples within two forcing periods, $l$ is the sampling index and the discrete time is $t_{l}=2 \pi l /(\Omega N) ; k=0,1 / 2,1,3 / 2$, etc. such that $\hat{q}_{0}, \hat{q}_{1 / 2}, \hat{q}_{1}$ give the base flow, subharmonic mode and fundamental mode, respectively. The growth rate and phase velocity of the fundamental mode are given by

$$
\begin{gathered}
\alpha_{i}(x)=-\frac{\operatorname{Re}_{\delta}}{\operatorname{Re}_{\infty}} \frac{1}{\hat{q}_{1}^{\max }} \frac{\partial \hat{q}_{1}^{\max }}{\partial x}, \\
c(x)=\operatorname{Re}_{\infty} F\left(\frac{\partial \phi_{1}}{\partial x}\right)^{-1},
\end{gathered}
$$

where $\phi_{k}=\arg \left(\hat{q}_{k}\right)$ is the phase angle of the perturbation and $F$ is the dimensionless frequency defined by (4.1).

The time integration is performed until the flow has reached a periodic solution. This is ensured by inspecting the subharmonic of the perturbation, whose amplitude is then at least one order of magnitude less than the fundamental perturbation.

\section{Flow cases and the laminar base flow}

Figure 1 shows an isobar at 80 bar (yellow solid line) in the $T-\vartheta$ diagram, together with the critical point (magenta square), pseudo-critical point (magenta pentagram), Widom line (white dashed line), saturation curves (blue on the liquid side and red on the vapour side) as well as a partition of the compressibility factor $Z=p^{*} /\left(\rho^{*} R^{*} T^{*}\right)$, which characterizes the non-ideality of the fluid. The pseudo-critical point is defined on the isobar where $C_{p}$ reaches its maximum, which helps to define the Widom line (Sciortino et al. 1997; Raju et al. 2017) and identify the point where non-ideal-gas effects are most prominent. As shown in the figure, close to the (pseudo-) critical 
point, the fluid shows large non-ideality where the compressibility factor $Z$ is far from 1.0 (less than 0.5, highlighted with the black background). On the other hand, the ideal-gas assumption can be made only when the compressibility factor is close to one, e.g. in the north-eastern part of the $T-\vartheta$ diagram. Along the isobar of 80 , the liquid-vapour phase boundary vanishes. Therefore, when the temperature increases from $220 \mathrm{~K}$, crosses the pseudo-critical point of $307.7 \mathrm{~K}$ and reaches $800 \mathrm{~K}$, the fluid undergoes a liquid-like to vapour-like transition and eventually approaches the ideal-gas regime, meanwhile, the thermodynamic and transport properties show large variations near the pseudo-critical point (see also appendix A). The temperatures highlighted on the isobar $(\mathrm{T} 220, \mathrm{~T} 240, \ldots)$ indicate the free-stream temperature that will be investigated in the following part of the paper.

Along the isobar shown in figure 1, we consider eight groups of cases, of which four are with subcritical free-stream temperatures (T220, T240, T260 and T280), and four are with supercritical temperatures (T320, T340, T360 and T800) in the free stream. For each group, four Eckert numbers are considered $\left(E c_{\infty}=0.05,0.10,0.15\right.$ and 0.20 ), leading to a total of 32 cases. On the upper half of table 1, we show the cases with subcritical $T_{\infty}^{*}$. The temperature profiles for these cases remain subcritical, except for the T280E4 case, which becomes transcritical $\left(T_{\infty}^{*} / T_{p c}^{*}<1\right.$ and $\left.T_{w}^{*} / T_{p c}^{*}>1\right)$ due to sufficient viscous heating. On the lower half, the supercritical cases are listed. Cases listed in table 1 are subjected to non-ideal-gas effects since they fall into the shaded area highlighted in figure 1, except for the T800 case, which serves as a reference for the ideal-gas regime. It can be anticipated that the T280 and T320 cases, which are closer to the Widom line, will have the most significant non-ideal-gas effects. It is also possible to notice that the free-stream Mach number remains subsonic in the subcritical cases and can be supersonic in the supercritical cases for the investigated range of Eckert numbers.

The base flow profiles are shown in figure 2 for cases T260, T280, T320 and T340. We show the distribution of temperature, density, viscosity and streamwise velocity as functions of the $\delta^{*}$-scaled wall-normal coordinate $y \cdot R e / R e_{\delta}$. In the temperature panel (a), the pseudo-critical point and the highly non-ideal regime around it are highlighted with a red line and yellow background. The flow temperature increases from the freestream value, reaching a maximum at the wall due to viscous heating. Compatible with table 1, the T280E4 case has crossed the Widom line which leads to a significant density drop from the liquid-like (subcritical) to the vapour-like (supercritical) regime, as shown in figure $2(b)$ (see also figure 23 ). The density gradients are comparably smaller for the other cases. We show the distribution of the viscosity in panel $(c)$; the value notably drops when the temperature increases from the subcritical regime. In the supercritical regime, the viscosity increases again with temperature. This can be seen in the zoom-in plot of case T340. As a result, the local Prandtl number $\mathrm{Pr}_{l}$, which is usually assumed constant in flows with the ideal-gas assumption, demonstrates large variations near the pseudo-critical point. The non-ideal-gas effects are also visible in the velocity distributions, shown in figure $2(d)$. In the subcritical and transcritical cases, the velocity develops a fuller profile with increasing Eckert number, which is opposite to what is observed for the supercritical and the ideal-gas cases, where the boundary-layer thickness increases with Eckert number.

Although the fluid can be highly non-ideal, the self-similar solution makes use of the constant pressure in the wall-normal direction, such that all the local variables can be solved as unary functions of $\eta$ (2.8) only. The base flow has been validated by comparing with the laminar results obtained with the DNS code (without perturbation) in appendix $\mathrm{B}$ in order to further verify the use of the self-similar solution with highly non-ideal fluids over adiabatic flat plates. 

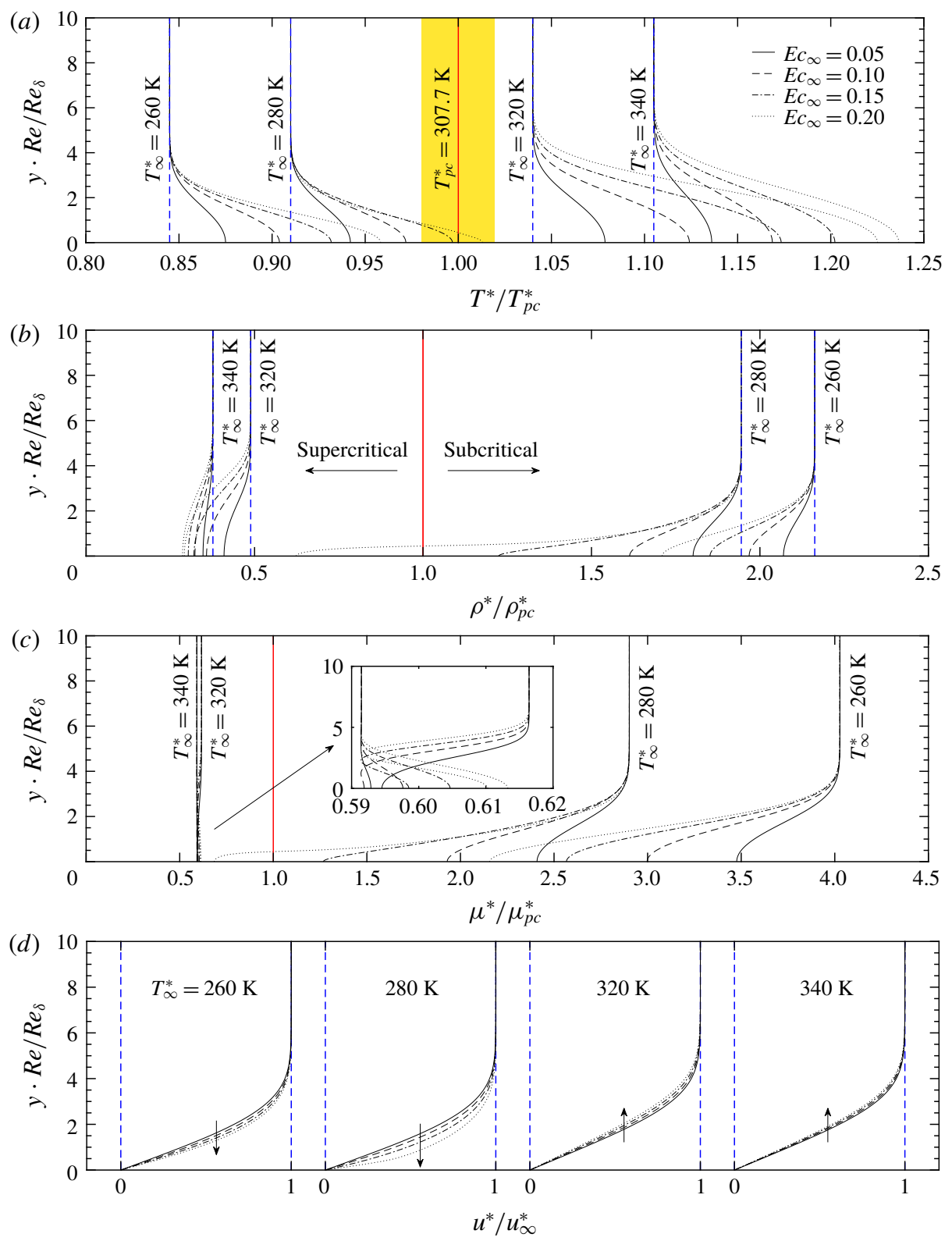

Figure 2. (Colour online) Base flow profiles of the T260, T280, T320 and T340 cases. Panels show the $(a)$ temperature, $(b)$ density $(c)$ viscosity and $(d)$ streamwise velocity as functions of the $\delta^{*}$-scaled wall-normal coordinate $y \cdot R e / R e_{\delta}$. The red solid line indicates the pseudo-critical point. The coloured area in $(a)$ schematically shows the regime where non-ideal-gas effects are most prominent. A zoom-in of the viscosity for cases T320 and T340 is also shown in $(c)$. Arrows in panel $(d)$ stand for the increase of the Eckert number $E c_{\infty}$ 


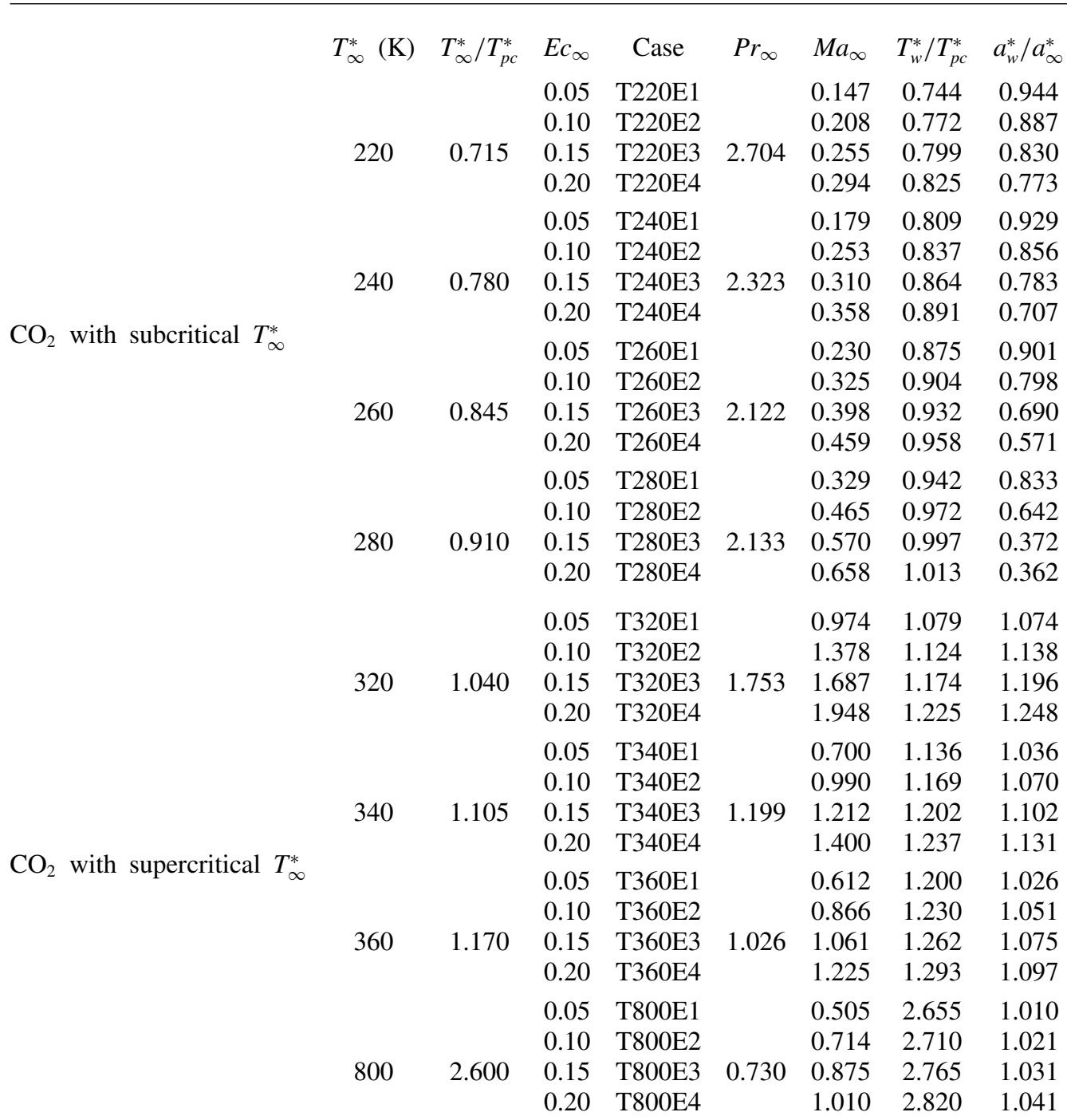

TABLE 1. Numerical parameters of the cases investigated. $T_{w}^{*}$ is the wall temperature, which results from the viscous heating and the adiabatic boundary condition. Note that to understand the transcritical regime, more cases have been investigated for $T_{\infty}^{*}=280 \mathrm{~K}$, in addition to the cases shown in this table.

\section{Results and discussions}

In this section, we analyse the stability of boundary-layer flows by discussing the neutral curve, growth rate, phase velocity, perturbation profiles as well as the inviscid equations. Two-dimensional perturbations are first studied (\$\$4.1-4.3), while oblique effects are discussed in $\S 4.4$. The dimensionless frequency $F$ is introduced,

$$
F=\frac{2 \pi F^{*} \mu_{\infty}^{*}}{\rho_{\infty}^{*} U_{\infty}^{* 2}}=\frac{\omega}{R e_{\delta}},
$$




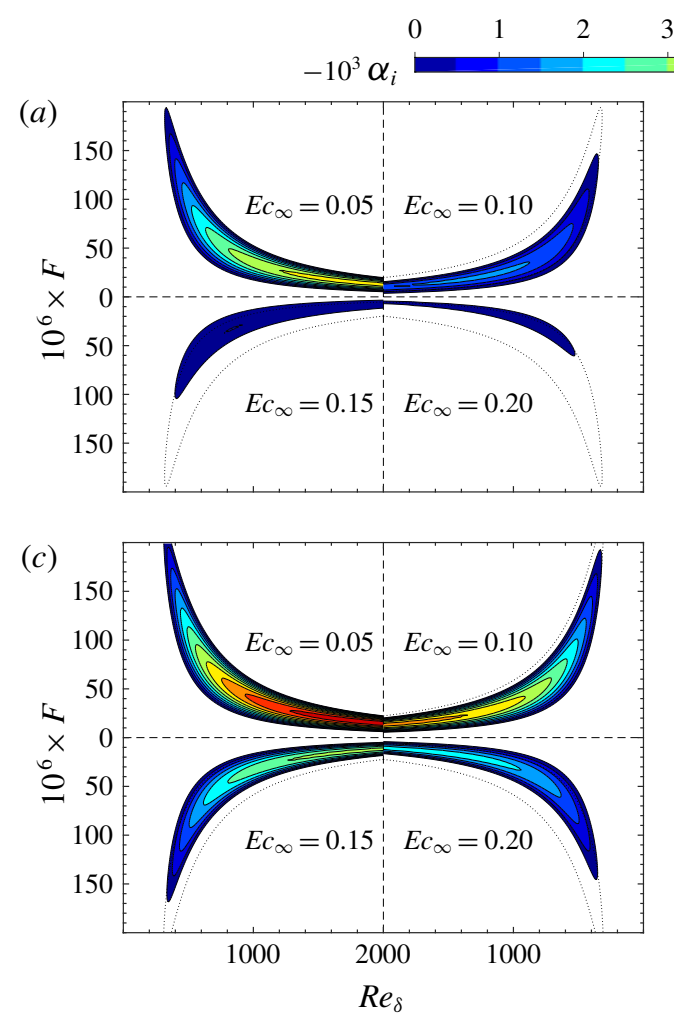

(b)

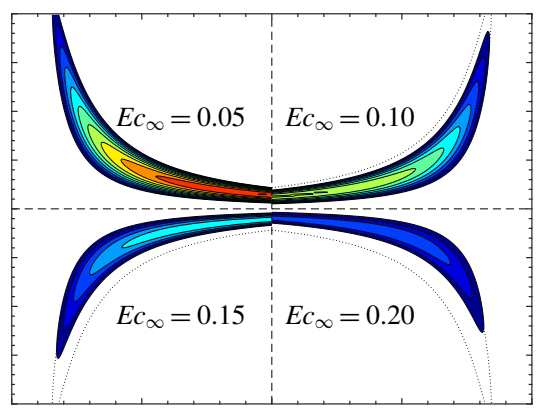

$(d)$

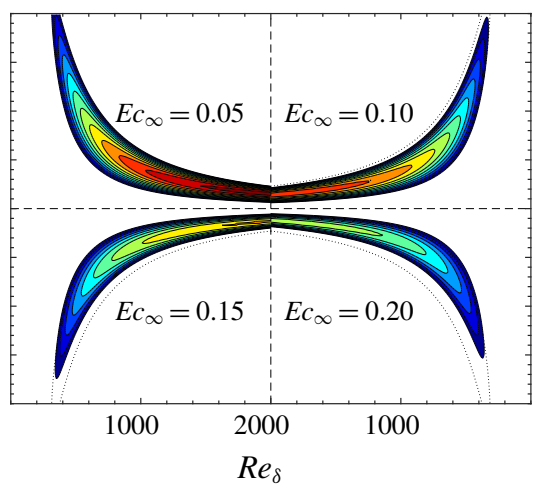

FIGURE 3. (Colour online) Growth rates $\left(-\alpha_{i}\right)$ of 2-D perturbations in the $F-R e_{\infty}$ stability diagram with supercritical free-stream temperatures: (a) $T_{\infty}^{*}=320 \mathrm{~K},(b) T_{\infty}^{*}=340 \mathrm{~K},(c)$ $T_{\infty}^{*}=360 \mathrm{~K}$ and $(d) T_{\infty}^{*}=800 \mathrm{~K}$. The coordinate in each panel has been mirrored in the centre in order to compare results for the four Eckert numbers.

which is directly proportional to the physical frequency $F^{*}$ of the perturbation, once the free-stream parameters are chosen.

\subsection{The supercritical regime}

Figure 3 shows the distribution of the local growth rate $\left(-\alpha_{i}\right)$ in the $F-R_{\delta}$ diagram. The contour levels start from the neutral curve $\left(\alpha_{i}=0\right)$, up to the largest growth rate in the range of $\operatorname{Re}_{\delta}$ and $F$ considered. In each panel the canvas is divided into four quadrants with mirrored coordinates to help compare results of four Eckert numbers. The dotted lines represent the neutral curve for $E c_{\infty}=0.05$, which are also plotted in the quadrants with the higher Eckert numbers to highlight the compressibility effects. Among the results, figure $3(d)$ provides a reference for the ideal-gas regime with $T_{\infty}^{*}=$ $800 \mathrm{~K}$, where it can be seen that an increase in Eckert (Mach) number stabilizes the flow, as the growth rate reduces and the extent of the neutral curve decreases. This is expected and has been documented in the past by Mack (1984).

However, if the free-stream temperature decreases towards values slightly above the pseudo-critical temperature, the non-ideal-gas effects considerably increase the stability of the base flow for high Eckert numbers. For example, the neutral curve for case T320E4 has become smaller, indicating a very narrow band of unstable frequencies. 


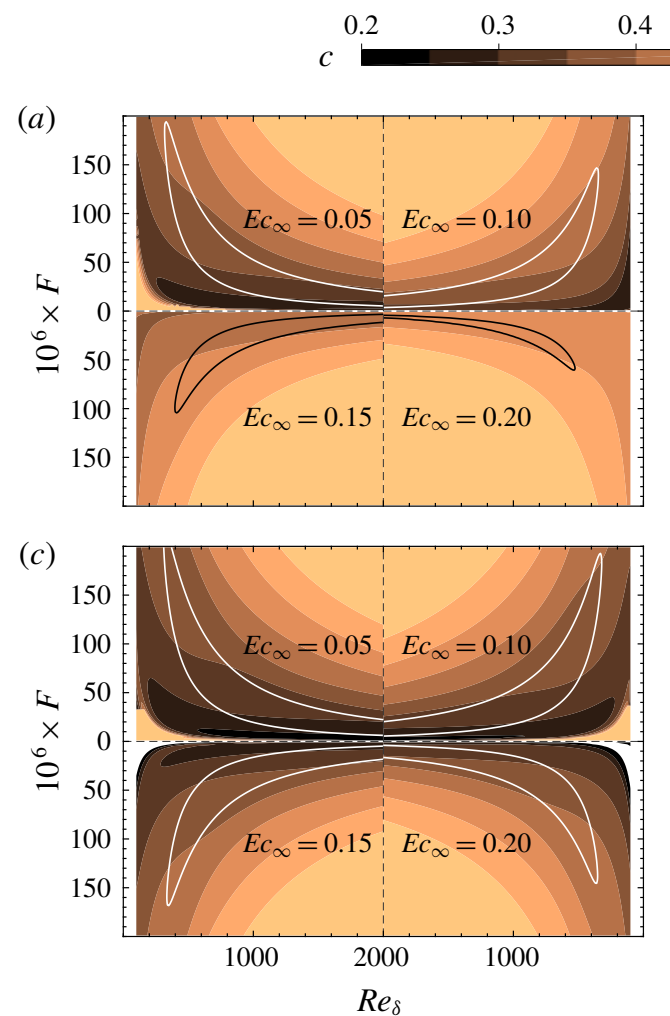

(b)

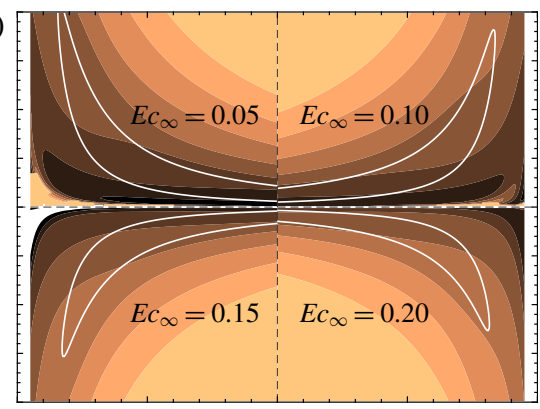

(d)

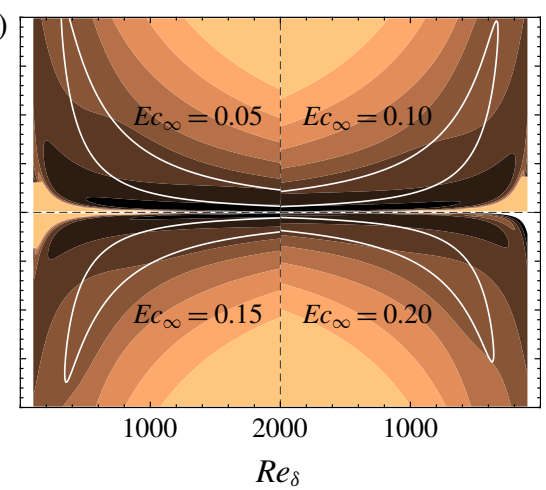

FIgURE 4. (Colour online) Phase velocities of perturbations (coloured contours) in the $F-R e$ stability diagram with supercritical free-stream temperatures: $(a) T_{\infty}^{*}=320 \mathrm{~K}$, (b) $T_{\infty}^{*}=340 \mathrm{~K},(c) T_{\infty}^{*}=360 \mathrm{~K}$ and $(d) T_{\infty}^{*}=800 \mathrm{~K}$. The solid lines indicate neutral curves of the corresponding cases.

For low Eckert numbers (e.g. $E c_{\infty}=0.05$ ), the size of the neutral curve is comparable, but the growth rate decreases with decreasing free-stream temperature.

Note that the results presented in figure 3 can be interpreted either by specifying $E c_{\infty}$ and comparing results for different $T_{\infty}^{*}$, or conversely setting $T_{\infty}^{*}$ and comparing different $E c_{\infty}$. Both show a stabilization of the base flow through non-ideal-gas (varying $T_{\infty}^{*}$ ) and compressibility effects (varying $E c_{\infty}$ ). One may argue that $M a_{\infty}$ can be used to show the compressibility effects. Here we clarify the advantage of using $E c_{\infty}$ instead of $M a_{\infty}$. From table 1, it is clear that a higher $E c_{\infty}$ corresponds to a higher $M a_{\infty}$, therefore the stabilization due to compressibility effects can be characterized by $M a_{\infty}$. However, $M a_{\infty}$ is not a suitable parameter to measure the degree of compressibility when non-ideal-gas effects are present. In fact, the speed of sound for the non-ideal gas drops sharply near the critical point. For example, for $\mathrm{CO}_{2}$ at 80 bar the speed of sound $a^{*}$ drops from 987.5 to $178.9 \mathrm{~m} \mathrm{~s}^{-1}$ and increases again to $433.3 \mathrm{~m} \mathrm{~s}^{-1}$ when temperature increases from $220 \mathrm{~K}$, to the pseudo-critical value of $307.7 \mathrm{~K}$ and further to $800 \mathrm{~K}$, respectively. As a result, non-ideal-gas effects cannot be correctly quantified at fixed $M a_{\infty}$.

The phase velocity $\left(c=\omega / \alpha_{r}\right)$ shown in figure 4 is another important physical variable that characterizes the perturbation. The corresponding neutral curves are shown to indicate the region of instability. It is clear that the phase velocities within 

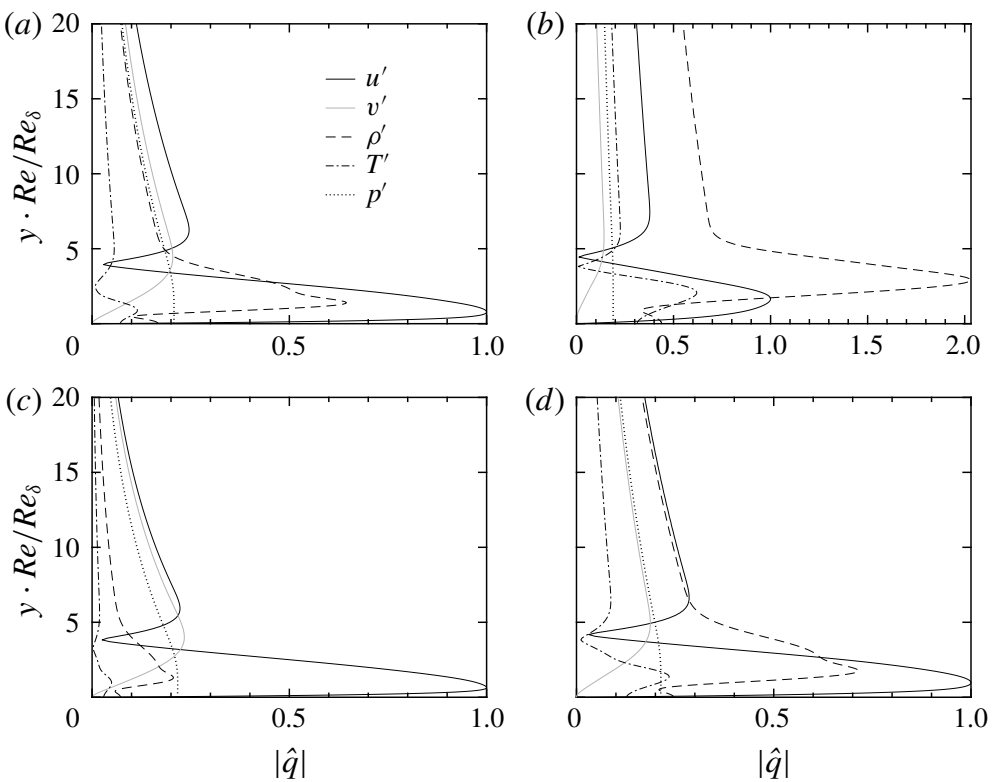

FIGURE 5. Profiles of the most amplified perturbations in the stability diagram of figure 4: (a) $T_{\infty}^{*}=320 \mathrm{~K}, E c_{\infty}=0.05,(b) T_{\infty}^{*}=320 \mathrm{~K}, E c_{\infty}=0.20$, (c) $T_{\infty}^{*}=360 \mathrm{~K}, E c_{\infty}=0.05$ and $(d) T_{\infty}^{*}=360 \mathrm{~K}, E c_{\infty}=0.20$.

the neutral curves remain between 0.2 and 0.6. The distributions of $c$ in the $F-R e_{\delta}$ diagram are similar for all the cases, i.e. an increase in $R_{\delta}$ or $F$ leads to a larger $c$. Non-ideal-gas effects, as well as compressibility effects, are seen to both increase the phase velocity. In accordance with an actual experimental/engineering set-up, the growth rate and phase velocity can be readily transferred into a physical $x^{*}-F^{*}$ diagram using (2.5) and (4.1).

We show profiles of perturbations in figure 5 for $T_{\infty}^{*}=320 \mathrm{~K}$ and $T_{\infty}^{*}=360$. The results are also compared for two Eckert numbers of 0.05 and 0.20 . The profiles correspond to the largest growth rate in the $F-R e_{\delta}$ diagram shown in figure 3 , and they are normalized by the amplitude of the streamwise velocity perturbation $\hat{u}$. As shown in the figure, $\hat{u}$ and $\hat{v}$ are similar in all four cases. Perturbations are dominated by $\hat{u}$ except the T320E4 case, in which $\hat{\rho}$ dominates the entire perturbation fields. Figure 5 demonstrates that compressibility (increase $E c_{\infty}$ ) and non-ideal-gas effects (reduce $T_{\infty}^{*}$ ) both increase the amplitude of density perturbations.

This can be explained by the balance of the linearized continuity equation,

$$
\underbrace{\left(\mathrm{i} \alpha u_{0}-\mathrm{i} \omega\right) \hat{\rho}}_{1}+\underbrace{\mathrm{i} \alpha \rho_{0} \hat{u}}_{2}+\underbrace{\frac{\mathrm{d} \rho_{0}}{\mathrm{~d} y} \hat{v}}_{3}+\underbrace{\rho_{0} \frac{\mathrm{d} \hat{v}}{\mathrm{~d} y}}_{4}=0 .
$$

When non-ideal and compressibility effects are insignificant, see figure $5(c)$, the density gradient of the base flow is weak and, consequently, $\hat{\rho}$ is small in amplitude. Therefore, equation (4.2) is mainly balanced by term 2 and term 4 , as shown in figure $6(c)$. Either reducing $T_{\infty}^{*}$ or increasing $E c_{\infty}$ results in an increase of $\mathrm{d} \rho_{0} / \mathrm{d} y$. This increase is fairly significant when $T_{\infty}^{*}$ is close to the pseudo-critical point 

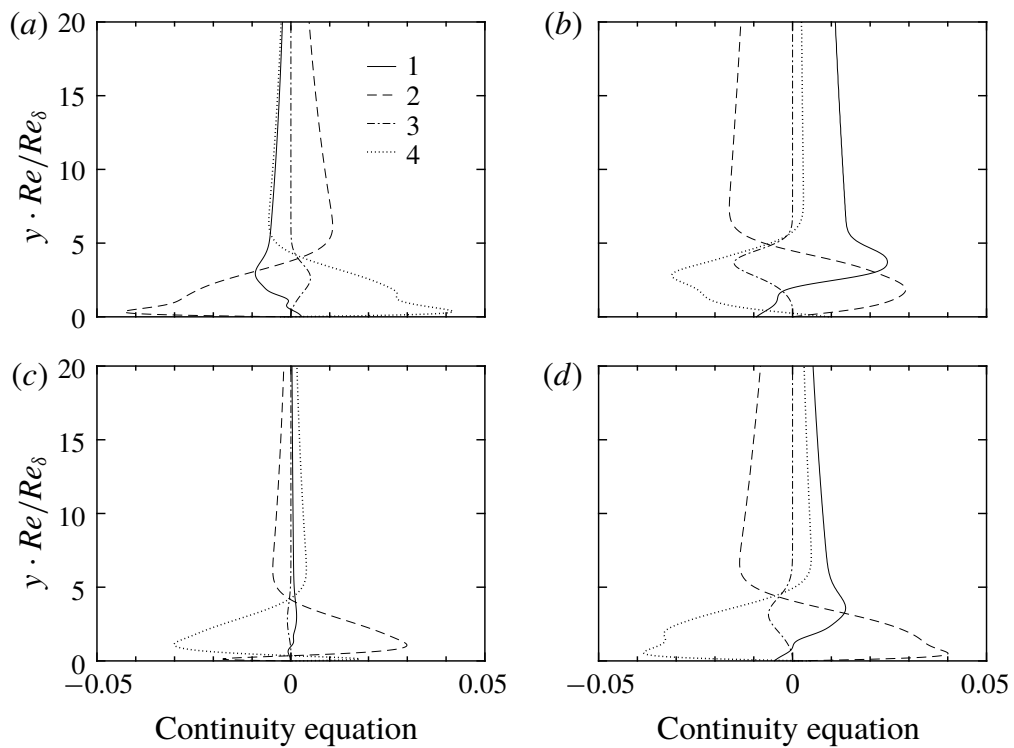

FIGURE 6. Balance of the continuity equation with the same parameters as in figure 5. Legend shows the four terms in (4.2).

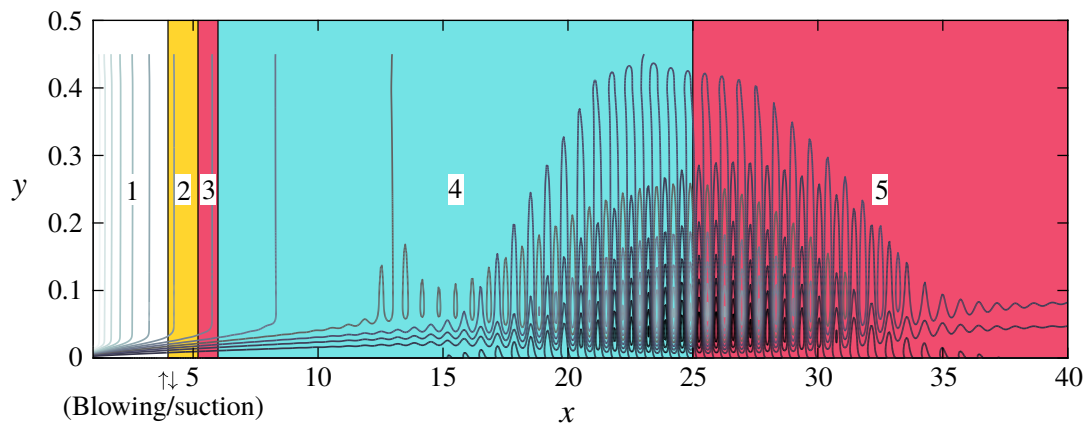

FIGURE 7. (Colour online) DNS of the T360E1 case. Contour lines of the wall-normal velocity in five coloured regions show the laminar flow (1), receptivity stage (2), modal decay before branch-I of the neutral curve (3), followed by the modal growth (4) and modal decay (5) after branch-II of the neutral curve. The up/down arrows at $x=4$ show the introduced wall blowing/suction. A movie of the perturbation development is available as a supplementary file (movie 1) at https://doi.org/10.1017/jfm.2019.348.

(see figure 2). The increase of term 3 has to be balanced with the help of term 1 , namely, a comparable density perturbation.

To account for possible non-parallel base flow effects and to validate the linear stability theory, DNS are performed for the T360 and T320 cases (the validation for the subcritical cases follows in the next section).

Figure 7 shows an example of the DNS results for the T360E1 case. Results are displayed by the contour plot of the wall-normal velocity, which nicely shows the development of the perturbation. Five physical regions can be identified: before the sinusoidal wall blowing/suction is introduced at $x=4$ to excite the T-S wave, the flow 
remains laminar in region 1 ; the receptivity process takes places in region 2 , where the forced external perturbation excites the T-S waves; the perturbation is well formed in the boundary layer and starts to follow the LST prediction in region 3. This region is yet ahead of branch-I of the neutral curve, therefore, perturbations goes through modal decay until region 4 ; in regions 4 and 5 , perturbations follow the linear stability theory and goes through a modal growth and decay.

To quantitatively compare LST and DNS, figure $8(a 1, b 1)$ shows the neutral curve in the $F-x$ diagram. The arrow in figure $8(a 2, b 2)$ indicates the location where wall blowing/suction is introduced (upstream of branch-I of the neutral curves). The amplitude of the forced blowing/suction has been kept small $\left(O\left(10^{-4}\right)\right.$ of the Mach number based on wall-normal momentum flux) so that the development of the perturbation stays in the linear regime. The frequencies $F=21 \times 10^{-6}$ and $31 \times 10^{-6}$ are chosen for the T320 and T360 cases respectively, which cut through the neutral curves (see figure $8 a 1, b 1$ ). One would expect modal growth of the perturbation between the two branches of each neutral curve.

The DNS is thoroughly compared with LST in figure $8(a 2, b 2, a 3, b 3)$ in terms of the growth rate and phase velocity. A local average is applied to the DNS results to remove numerical oscillations. For the T360 cases (b1-b3), the DNS and LST match very well. Small differences can be seen for the high Eckert number cases, which can be attributed to the lower growth rate and the ensuing lower amplitude of the perturbation. To calculate the growth rate (2.14), a lower amplitude of perturbations can cause a relatively larger error.

On the other hand, the flow is considerably stabilized at $T_{\infty}^{*}=320 \mathrm{~K}$ (figure $8 a 1$ $a 3$ ). As can be inferred from the neutral curve (figure $8 a 1$ ), the unstable region in $x$ is substantially reduced. Figure $8(a 2, a 3)$ shows that the DNS and LST match well at $E c_{\infty}=0.05$. The difference becomes larger with increasing Eckert number. In the two cases of $E c_{\infty}=0.15$ and 0.20 , the growth rate predicted by LST is very small, which is not completely captured by DNS. Note that we have shortened the domain of the DNS for the $E c_{\infty}=0.15$ and 0.20 cases, the number of grid points both in $x$ and $y$ have been refined to make sure the results are grid independent. Differences in the phase velocity at $E c_{\infty}=0.20$ also indicate that the modal instability predicted by LST (though the growth rate is small but positive) cannot be well captured in the DNS simulations. One of the possible reasons lies in the fact that in DNS the perturbed flow field is a result of multiple eigenmodes. Therefore, when the growth rate of the single unstable mode is exceedingly small, the flow field can be co-dominated by multiple decaying modes, and the post-processed growth rate and phase velocity from DNS cannot match a single mode from LST.

The above results have confirmed that the non-ideal-gas effects stabilize the flow in the supercritical regime. The stabilization is more significant when the free-stream temperature is closer to the pseudo-critical value and/or when coupled with strong compressibility effects.

\subsection{The subcritical and transcritical regimes}

In this section, we discuss cases with subcritical free-stream temperatures (T220, T240, T260 and T280). Recall the base flow in $\S 3$, the laminar flow stays in the subcritical regime except for the transcritical case with $T_{\infty}^{*}=280 \mathrm{~K}$ and $E c_{\infty}$ sufficiently large (T280E4).

The growth rates for these cases are shown in figure 9. Comparing the four quadrants in each panel, it can be seen that the flow is stabilized by increasing $E c_{\infty}$ 
$(a 1)$

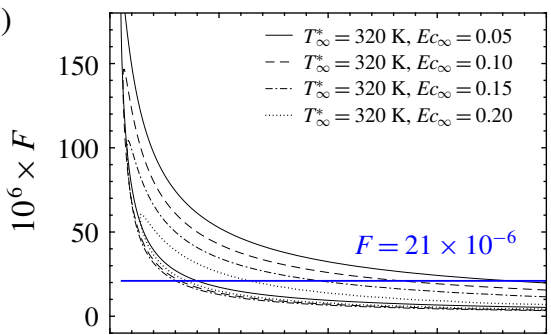

(a2)

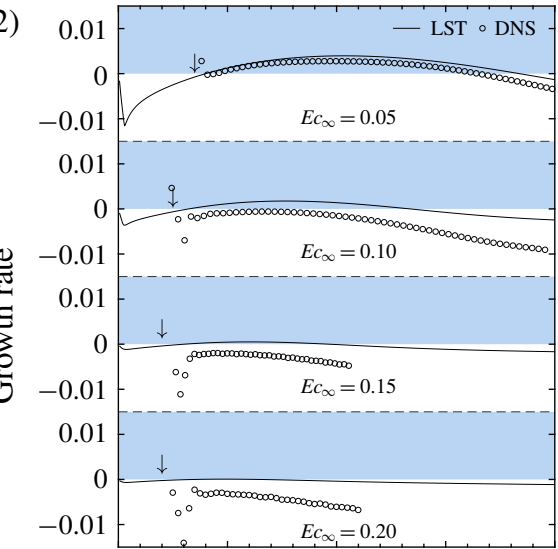

(a3)

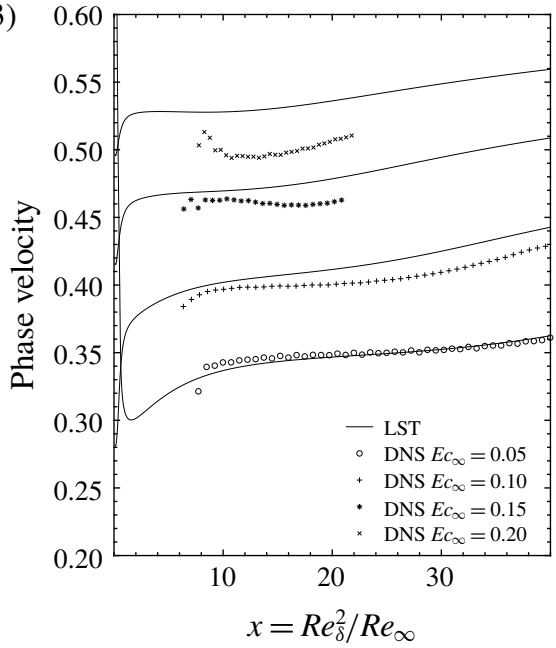

(b1)

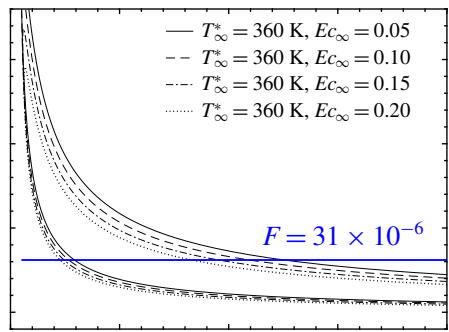

(b2)

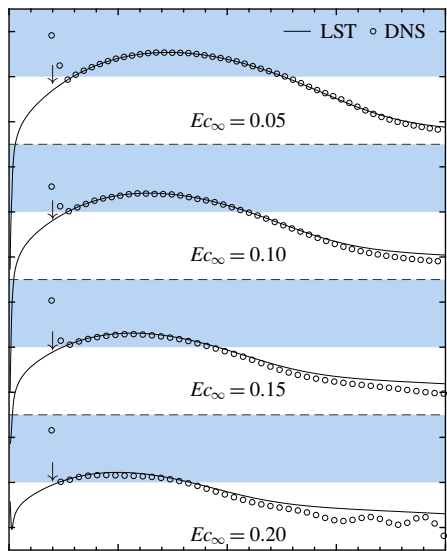

(b3)

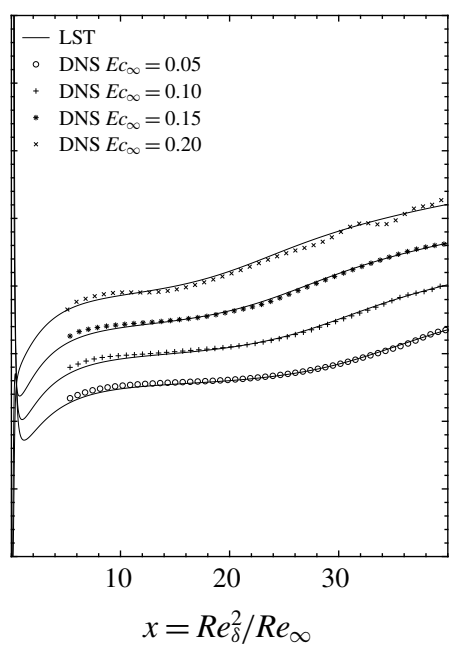

FIGURE 8. (Colour online) DNS validation of the T320 (a1-a3) and T360 (b1-b3) cases. $(a 1)$ and $(b 1)$ show the neutral curve in the $F-x$ diagram. The blue solid line indicates the frequency of wall blowing/suction introduced to excite the T-S wave. (a2), (b2), (a3) and (b3) provide comparisons of the growth rate and phase velocity between DNS and LST. The arrows in $(a 2)$ and $(b 2)$ indicate the position where wall blowing/suction is introduced.

in the subcritical regime. Similar to the supercritical regime, non-ideal-gas effects (increase in $T_{\infty}^{*}$ ) further stabilize the subcritical flows. In the subcritical regime, the perturbation profiles for cases T240E1, T240E4, T280E1 and T280E3 are provided in figure 10. Recalling the discussion for the supercritical regime, the profiles in 

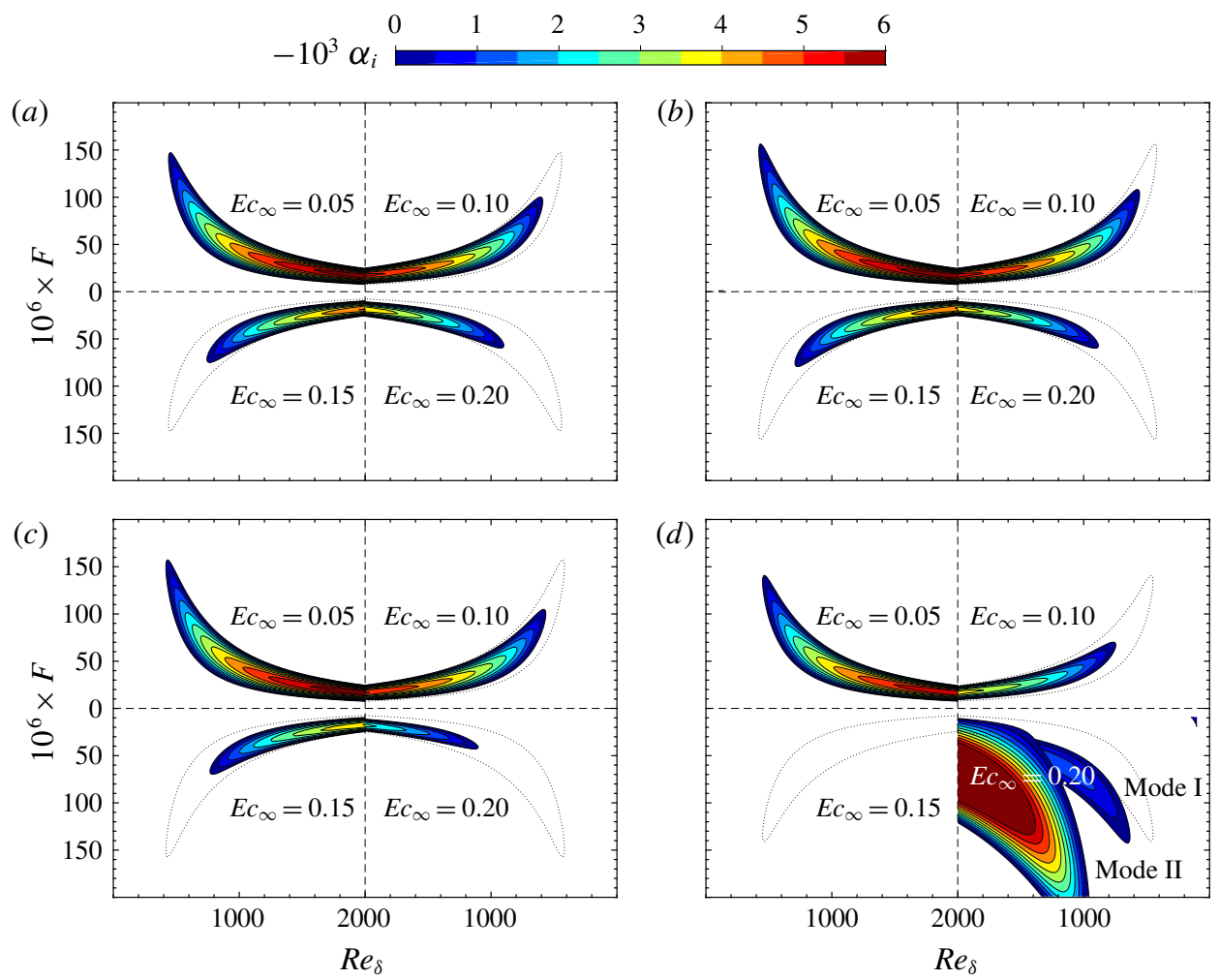

(d)

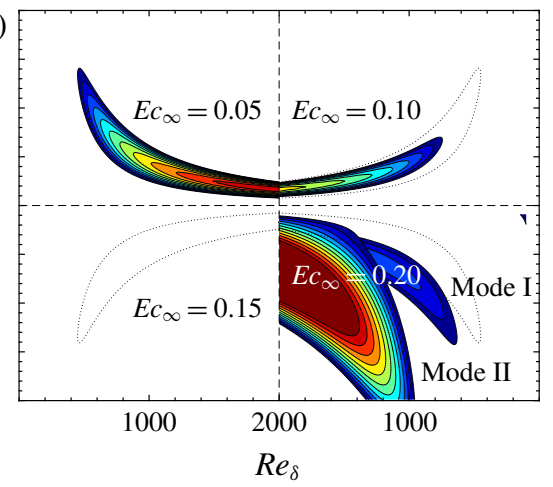

FIGURE 9. (Colour online) Growth rates of perturbations in the $F-R e_{\delta}$ stability diagram with subcritical free-stream temperatures: $(a) T_{\infty}^{*}=220 \mathrm{~K},(b) T_{\infty}^{*}=240 \mathrm{~K},(c) T_{\infty}^{*}=$ $260 \mathrm{~K}$ and $(d) T_{\infty}^{*}=280 \mathrm{~K}$.

the subcritical regime follow a similar trend: the compressibility and non-ideal-gas effects increase the amplitude of the density perturbation due to the increase of the base flow's density gradient $\partial \rho_{0} / \partial y$ and the balancing mechanism of the linearized continuity equation. To avoid repetition, the quantitative DNS validation of the stabilization of non-ideal-gas effects in the subcritical regime has been provided in appendix C.

More attention shall be paid to the T280 cases in figure $9(d)$. The stability of the base flow noticeably increases with growing Eckert number. For instance, at $E c_{\infty}=$ 0.15 no modal instability can be seen for Reynolds numbers up to $R e_{\delta}=2000$. After the base flow crosses the pseudo-critical point (at $E c_{\infty}=0.20$ ), a co-existence of dual unstable modes is observed, which we term Mode I and Mode II. The neutral curve of Mode I has a similar shape to the subcritical regime, while Mode II has a much larger growth rate and unstable band. For this case, a typical eigenspectrum is provided in figure 11 with $R e_{\infty}=1500$ and $F=45 \times 10^{-6}$. As it can be clearly inferred, both modes are unstable with the eigenvalue of $\alpha=0.1338-0.001293 i$ (Mode I) and $\alpha=$ $0.2037-0.001887 i$ (Mode II). As such, it can be concluded that with increasing Eckert number, the non-ideal-gas effects initially stabilize Mode I. However, after the base flow becomes transcritical, Mode II emerges and finally dominates the instability.

To further explore the transcritical regime for the T280 cases, we show in figures 12 and 13 the detailed evolution of the growth rate and phase velocity on gradually 


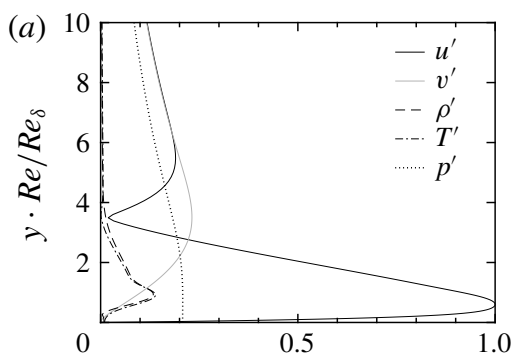

(b)
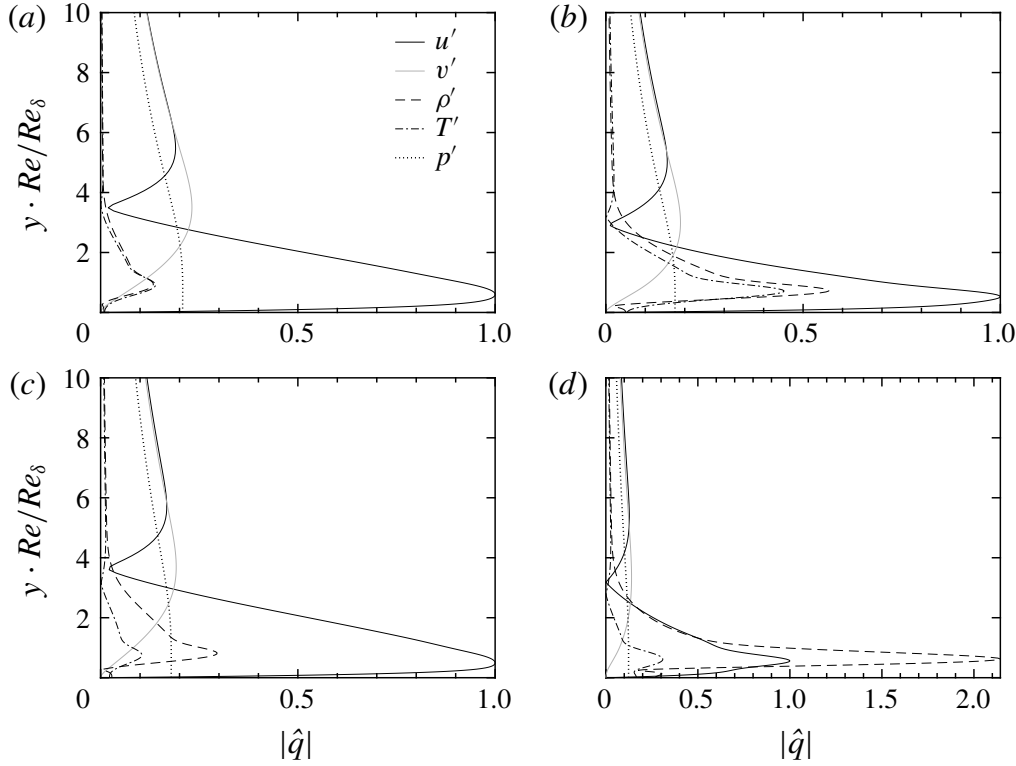

FIgURE 10. Profiles of the most amplified perturbations in the stability diagram of figure 9: (a) $T_{\infty}^{*}=240 \mathrm{~K}, E c_{\infty}=0.05$, (b) $T_{\infty}^{*}=240 \mathrm{~K}, E c_{\infty}=0.20$, (c) $T_{\infty}^{*}=280 \mathrm{~K}$, $E c_{\infty}=0.05$ and $(d) T_{\infty}^{*}=280 \mathrm{~K}, E c_{\infty}=0.15$.

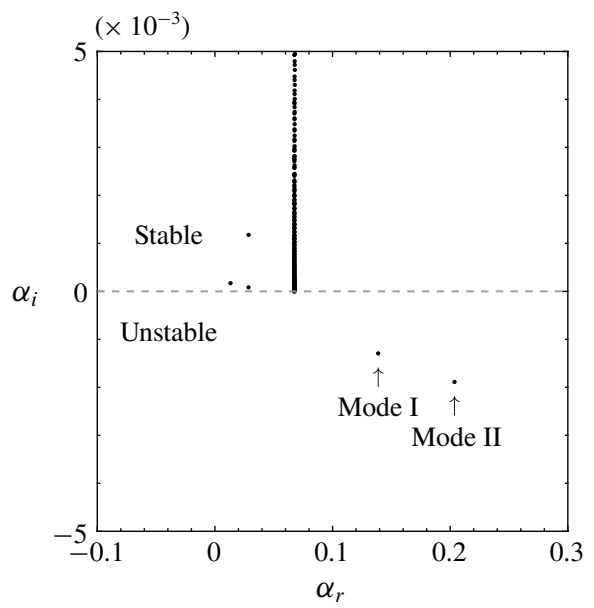

FIGURE 11. Eigenspectrum of the transcritical case with $T_{\infty}^{*}=280 \mathrm{~K}, E c_{\infty}=0.20, R e_{\delta}=$ 1500 and $F=45 \times 10^{-6}$. The dual modes are highlighted with arrows.

increasing the Eckert number from $E c_{\infty}=0.11$ to $E c_{\infty}=0.202$. In order to show the compelling stabilizing effect as observed in figure $9(d)$ (at $E c_{\infty}=0.15$ ), the range of $R e_{\delta}$ has been extended to $R e_{\delta}=4000$. Figure 12(a,b) shows the neutral curve of Mode I, while in figure $12(c)$ we show Mode II that becomes unstable at $E c_{\infty} \geqslant 0.19$. It appears that the maximum critical Reynolds number $R e_{\delta}$ occurs at $E c_{\infty}=0.16$. The flow enters the transcritical regime (the temperature crosses the pseudo-critical point) at $E c_{\infty} \geqslant 0.17$, and the growth rate and the extent of the neutral curve of Mode I again 
(a)

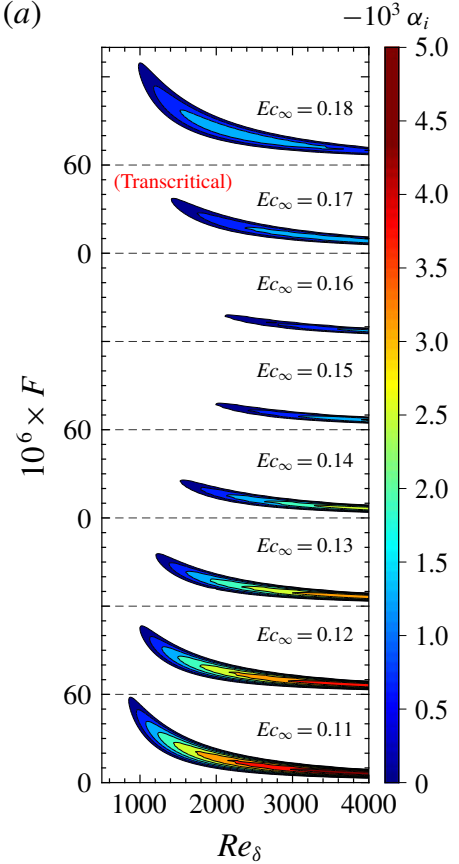

(b)

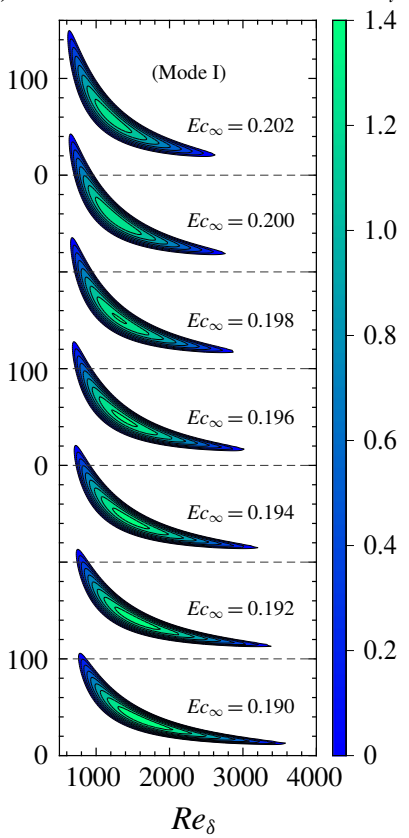

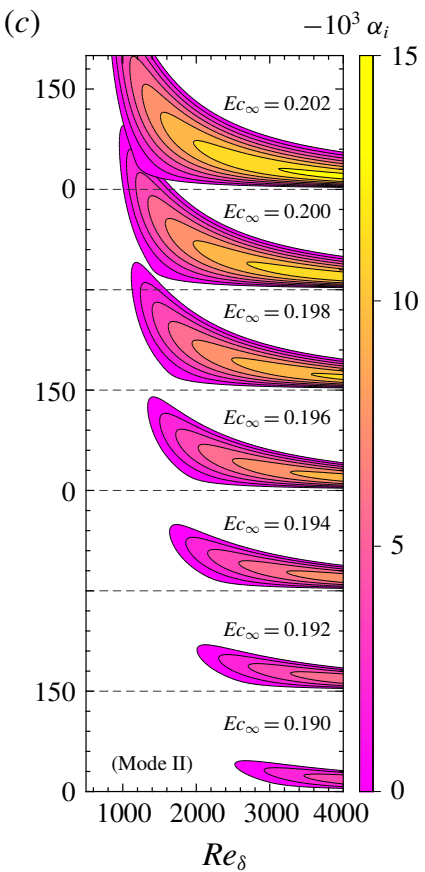

FIGURE 12. (Colour online) Growth rates of perturbations in the $F-R e_{\delta}$ stability diagram with $T_{\infty}^{*}=280 \mathrm{~K}$. (a) $E c_{\infty}=0.11,0.12, \ldots, 0.19$, (b) $E c_{\infty}=0.190,0.192, \ldots, 0.202$ (Mode I), (c) $E c_{\infty}=0.190,0.192, \ldots, 0.202$ (Mode II).

increase. Figure 12(b) shows the evolution of Mode I in the transcritical regime. With an increase in $E c_{\infty}$, the range of unstable $R e_{\delta}$ decreases, while the range for unstable $F$ increases. Most noteworthy is the growth rate of Mode II, shown in figure 12(c), which increases much faster with $E c_{\infty}$ and becomes much larger than Mode I. This indicates that the flow in the transcritical regime is significantly destabilized by nonideal-gas effects through Mode II. In addition, we also show the development of the phase velocity in figure 13. The phase velocity of Mode I remains between 0.2 and 0.54 , while Mode II is between 0.3 and 0.35. Both increase with Eckert number.

Similar to the super- and subcritical cases discussed before, we also use DNS to validate the results from LST and to confirm the co-existence of the dual modes, as well as the dominance of Mode II. We show the neutral curve for $E c_{\infty}=0.20$ (case T280E4) in the $F-x$ diagram in figure 14 . Mode II has a much larger unstable region both in terms of $F$ and $x$. In order to properly observe the two modes, the flow is perturbed with frequencies of $F_{1}=15 \times 10^{-6}$ and $F_{2}=75 \times 10^{-6}$, respectively. From the LST prediction in figure 14, the forced frequency $F_{1}$ shall excite Mode II on entering the neutral curve, and the perturbation of frequency $F_{2}$ will be modulated by Mode I and II sequentially.

Figure 15 shows the DNS validation, where the growth rate and phase velocity are compared with LST. In the $F_{1}$ case, the perturbation grows due to the positive growth rate of Mode II. In the $F_{2}$ case, the instability is initially dominated by Mode I and sequentially by Mode II, due to the cross-over of the growth rates of the two modes as shown in figure $15(c)$. In both cases, the DNS matches well with the LST predictions.

The perturbation profiles with $F=75 \times 10^{-6}$ obtained from LST and DNS are provided and compared in figure 16. The comparison is made at $x=16.00$ (Mode I 
(a)

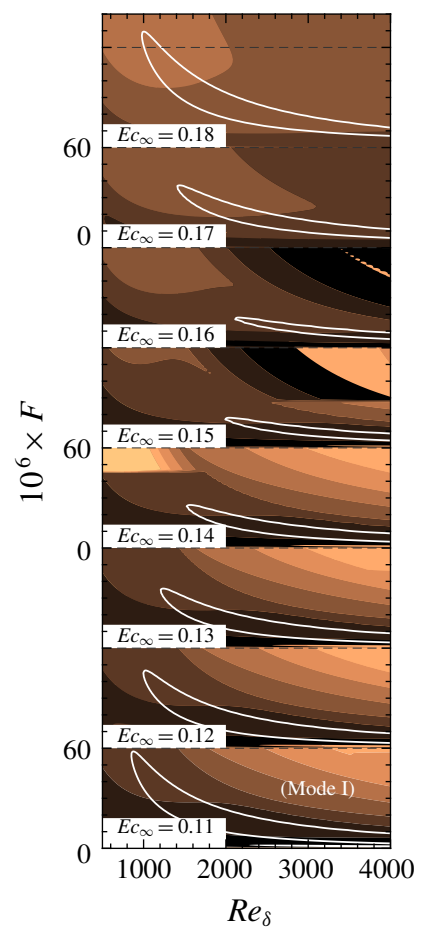

(b)

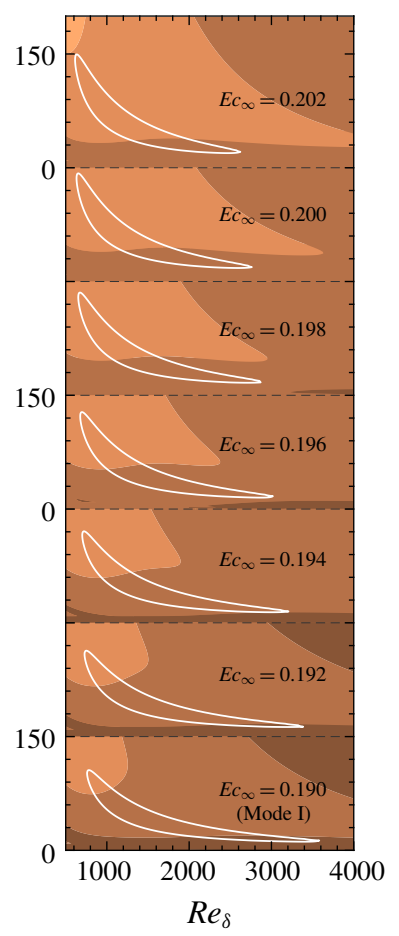

(c)

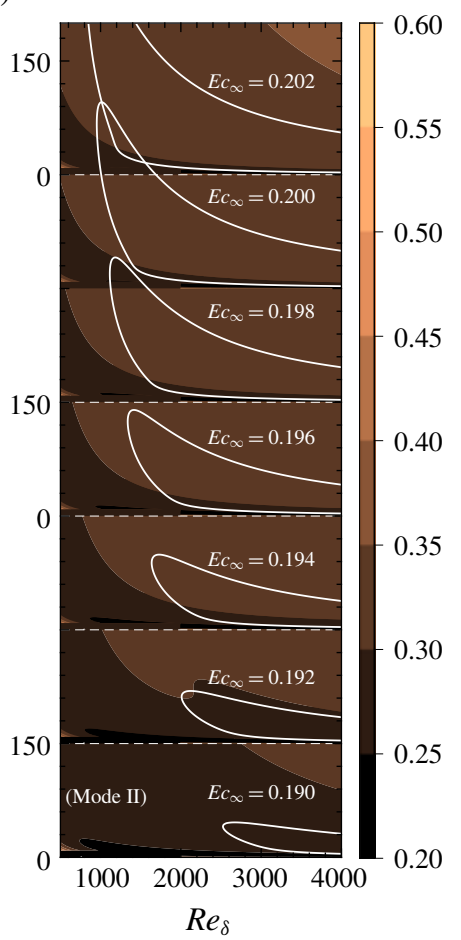

FIgURE 13. (Colour online) Phase velocities of perturbations in the $F-R e_{\delta}$ stability diagram with $T_{\infty}^{*}=280 \mathrm{~K}$. (a) $E c_{\infty}=0.11,0.12, \ldots, 0.19$, (b) $E c_{\infty}=0.190$, $0.192, \ldots, 0.202$ (Mode I), (c) $E c_{\infty}=0.190,0.192, \ldots, 0.202$ (Mode II).

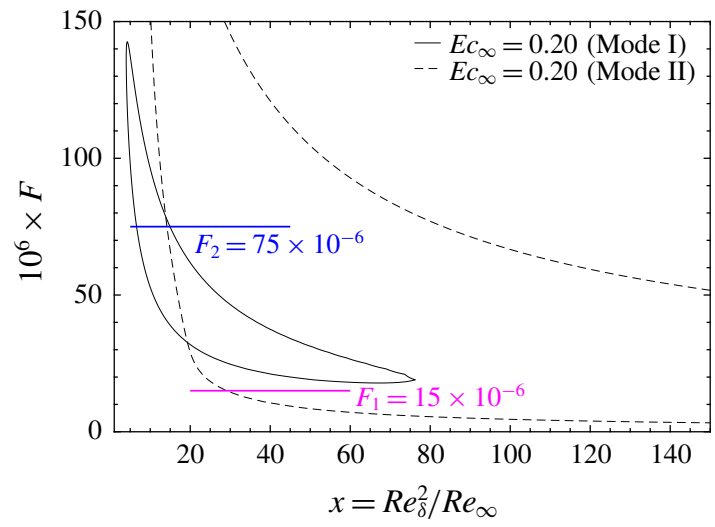

FIgURE 14. (Colour online) Neutral curve of the T280E4 case in the $F-x$ diagram. The magenta and blue lines indicate the domain and frequency $F_{1}=15 \times 10^{-6}$ and $F_{2}=75 \times$ $10^{-6}$ simulated in the DNS.

dominates) and $x=36.00$ (Mode II dominates). Figure 16 shows that the perturbation profiles obtained from DNS match very well with the LST predictions of Mode I and Mode II at $x=16.00$ and $x=36.00$, respectively. Due to the large gradient of $\partial \rho_{0} / \partial y$, 

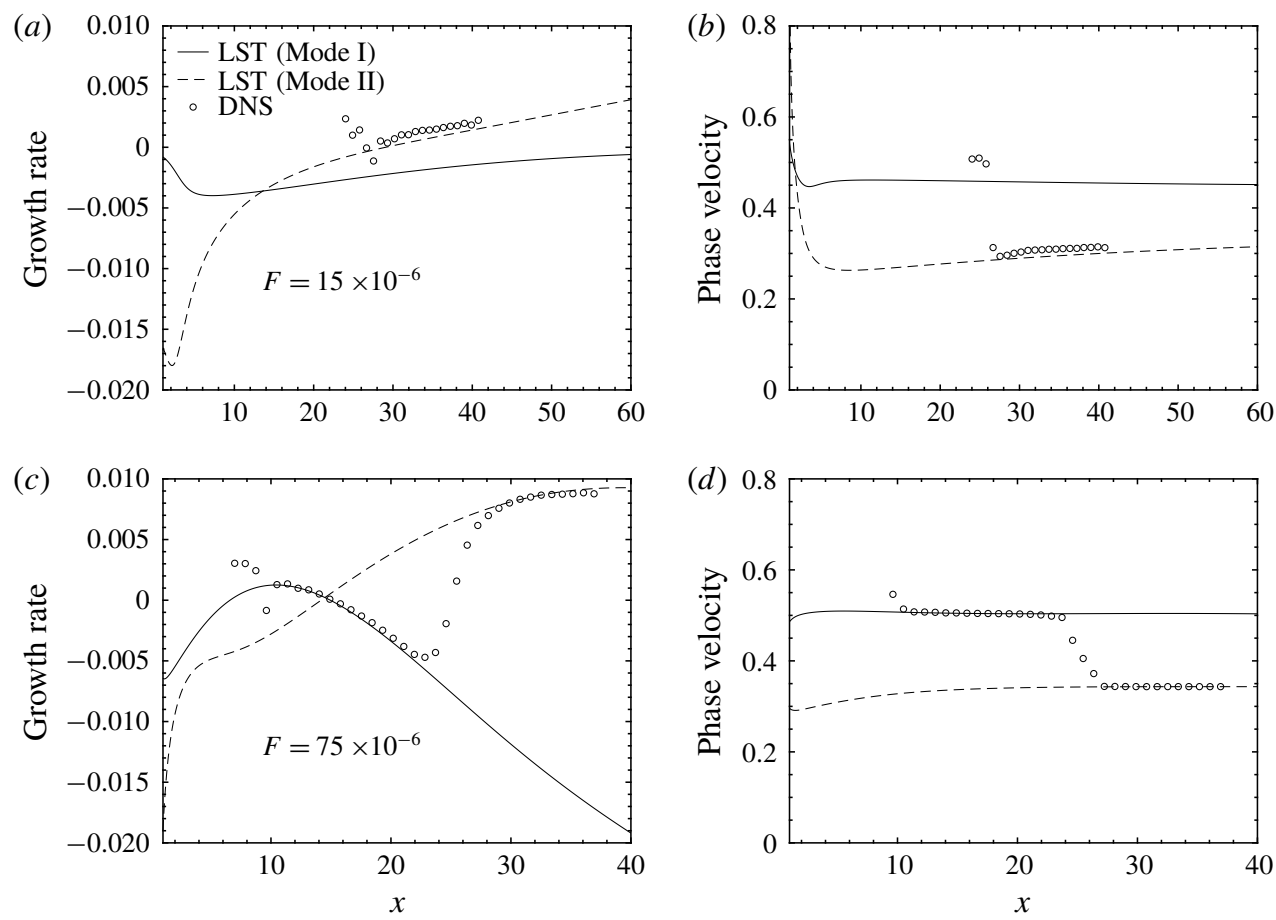

FIGURE 15. Comparison of the growth rate and phase velocity between LST and DNS: $T_{\infty}^{*}=280 \mathrm{~K} ; E c_{\infty}=0.20,(a, b) F=15 \times 10^{-6} ;(c, d) F=75 \times 10^{-6}$.
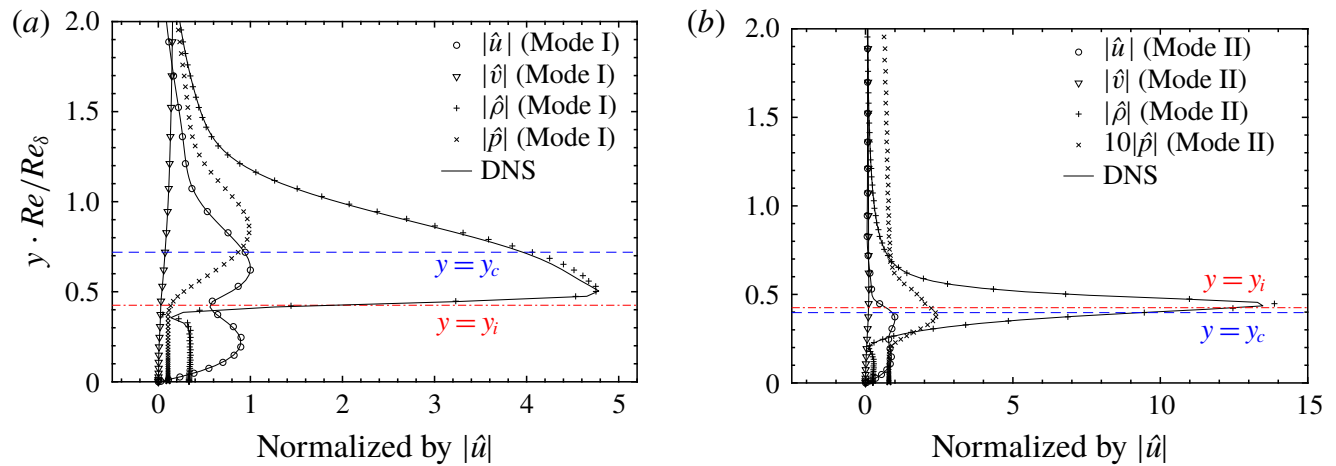

FIgURE 16. (Colour online) Comparison of the profiles $(\hat{u}, \hat{v}, \hat{\rho})$ of the perturbations between LST and DNS. The amplitude has been normalized by $|\hat{u}|: T_{\infty}^{*}=280 \mathrm{~K}, E c_{\infty}=$ $0.20, F=75 \times 10^{-6}$; (a) $x=16.00, R_{\delta}=1264.9$; (b) $x=36.00, R e_{\delta}=1894.7$. The critical point $\left(y=y_{c}, u_{0}\left(y_{c}\right)=c\right)$ and the generalized inflection point $\left(y=y_{i}\right)$ are denoted with the blue dashed line and red dash-dotted line respectively.

the density perturbation is the largest component for both modes. Note that $|\hat{\rho}|$ is considerably larger for Mode II. On the other hand, the velocity components $\hat{u}$ and $\hat{v}$ remain similar for both modes, indicating that Mode II is caused by the dramatic variation of thermodynamic and transport properties. The above comparisons validated 
the co-existence of the dual modes and the dominance of Mode II in the transcritical regime.

\subsection{Is Mode II comparable to Mack's second mode?}

Based on the findings in $\$ 4.2$, Mode II is generated when the fluid is transcritical although the flow is subsonic. In this section, using the inviscid theory, we clarify the relation to Mack's second mode which appears in hypersonic flows.

The inviscid stability equations for the 2-D perturbations are given by

$$
\left.\begin{array}{c}
\left(\alpha u_{0}-\omega\right) \hat{\rho}+\alpha \rho_{0} \hat{u}=\mathrm{i} \rho_{0} D \hat{v}+\mathrm{i} \frac{\partial \rho_{0}}{\partial y} \hat{v} \\
\alpha \hat{p}+\left(\alpha u_{0}-\omega\right) \rho_{0} \hat{u}=\mathrm{i} \rho_{0} \frac{\partial u_{0}}{\partial y} \hat{v} \\
\left(\alpha u_{0}-\omega\right) \rho_{0} \hat{v}-\mathrm{i} D \hat{p}=0 \\
\left(\alpha u_{0}-\omega\right) \rho_{0} \hat{e}+\alpha p_{0} \hat{u}=\mathrm{i} p_{0} D \hat{v}+\mathrm{i} \rho_{0} \frac{\partial e_{0}}{\partial y} \hat{v}
\end{array}\right\}
$$

where $e_{0}$ and $\hat{e}$ are the internal energy and its perturbation. Equation (4.3) can be reduced to a single equation,

$$
\begin{aligned}
& {\left[\left(\left.\frac{\partial \rho_{0}}{\partial e_{0}}\right|_{p} \frac{p_{0}}{\rho_{0}^{2}}-1\right) \alpha^{2}+\left.\left(\alpha u_{0}-\omega\right)^{2} \frac{\partial \rho_{0}}{\partial p_{0}}\right|_{e}\right]_{p}-\left(\left.\frac{\partial \rho_{0}}{\partial e_{0}}\right|_{p} \frac{p_{0}}{\rho_{0}^{2}}-1\right) D^{2} \hat{p}} \\
& +\left[\left.\frac{\partial \rho_{0}}{\partial e_{0}}\right|_{p} \frac{p_{0}}{\rho_{0}^{3}} \frac{\partial \rho_{0}}{\partial y}+\left(\left.\frac{\partial \rho_{0}}{\partial e_{0}}\right|_{p} \frac{p_{0}}{\rho_{0}^{2}}-1\right) \frac{2 \alpha}{\alpha u_{0}-\omega} \frac{\partial u_{0}}{\partial y}-\left.\frac{1}{\rho_{0}} \frac{\partial \rho_{0}}{\partial e_{0}}\right|_{p} \frac{\partial e_{0}}{\partial y}\right] D \hat{p}=0 .
\end{aligned}
$$

Following Mack (1984), neglecting the term related to $D \hat{p}$ results in

$$
D^{2} \hat{p}-\alpha^{2}\left[1-\left.\frac{\left(u_{0}-c\right)^{2}}{1-\left.\frac{\partial \rho_{0}}{\partial e_{0}}\right|_{p} \frac{p_{0}}{\rho_{0}^{2}}} \frac{\partial \rho_{0}}{\partial p_{0}}\right|_{e}\right] \hat{p}=0 .
$$

We introduce the relative Mach number $M_{r}$,

$$
M_{r}=\frac{u_{0}-c}{\sqrt{\left(1-\left.\frac{\partial \rho_{0}}{\partial e_{0}}\right|_{p} \frac{p_{0}}{\rho_{0}^{2}}\right)\left(\left.\frac{\partial \rho_{0}}{\partial p_{0}}\right|_{e}\right)^{-1}}} .
$$

Using Maxwell relations for partial derivatives of thermodynamic properties, it can be mathematically proven (see appendix D) that the relative Mach number (4.6) for non-ideal gases equals to

$$
M_{r}=\frac{u_{0}-c}{\sqrt{\left.\frac{\partial p_{0}}{\partial \rho_{0}}\right|_{s}}}=\frac{u_{0}-c}{a_{0}},
$$




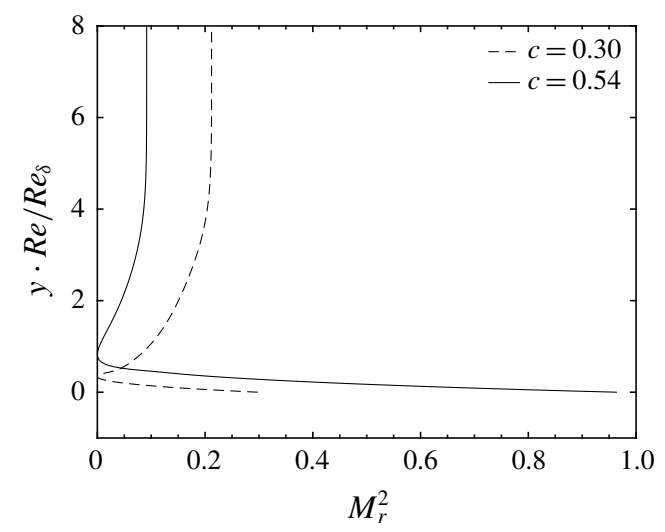

FIgURE 17. The relative Mach number with $T_{\infty}^{*}=280 \mathrm{~K}$ and $E c_{\infty}=0.20$.

which is equivalent to the result of Mack (1984). Equation (4.5) changes its behaviour from elliptic to hyperbolic when $\left(1-M_{r}^{2}\right)$ changes sign (from positive to negative) and multiple solutions (modes) can be present. This has successfully explained Mack's second mode in high-speed flows of ideal gas. Equation (4.7) indicates that the relative Mach number for non-ideal gases possesses the same physical nature, i.e. a local supersonic region relative to the phase velocity can give rise to multiple modes. We show in figure 17 the distribution of $M_{r}^{2}$. The results are given for phase velocities $c=0.30$ and 0.54 , which correspond to the lower and upper limits of the dual modes $\left(0.19 \leqslant E c_{\infty} \leqslant 0.20\right)$. The figure shows that $M_{r}$ stays always below 1 in the transcritical regime $\left(T_{\infty}^{*}=280 \mathrm{~K}\right.$ and $\left.E c_{\infty}=0.20\right)$ where the dual modes co-exist. This implies that Mode II is different from Mack's second mode.

Another evidence to highlight the difference between Mode II and Mack's second mode is the co-existence of dual unstable modes with same parameters $\left(F, \beta, \operatorname{Re}_{\delta}\right.$ and $\left.E c_{\infty}\right)$. In fact, according to the new terminology summarized in Fedorov \& Tumin (2011), Mack's second mode is a result of synchronization between the fast mode and the slow mode, which stems from the continuous spectrum of the fast and slow acoustic waves. As such, only one unstable eigenmode is present for certain parameters $\left(F, \beta, R e_{\delta}\right.$ and $\left.E c_{\infty}\right)$ of the base flow and the perturbation. This leads to the conventional neutral curve for hypersonic boundary-layer flows (see for example figure 1 in Ren, Fu \& Hanifi 2016). However, the neutral curves of Mode I and Mode II presented here, indeed overlap with each other. Both modes can be unstable with the same frequency $F$ and wavenumber.

Under the inviscid assumption, Lees \& Lin (1946) derived the generalized inflection point criterion $D\left(D u_{0} / T_{0}\right)$, which gives the necessary condition for a compressible boundary-layer flow to be inviscidly unstable. They used the ideal-gas equation of state $\left(\rho_{0} T_{0}=1\right)$ and below we show that a similar criterion applies for non-ideal gases. From (4.4) for $\hat{p}$ and using the relation $D \hat{p}=-\mathrm{i} \rho_{0}\left(\alpha u_{0}-\omega\right) \hat{v}$, the equation for $\hat{v}$ then reads,

$$
\left(\alpha^{2}+\theta\right) \hat{v}+\xi D \hat{v}+\eta D^{2} \hat{v}=0
$$

where 


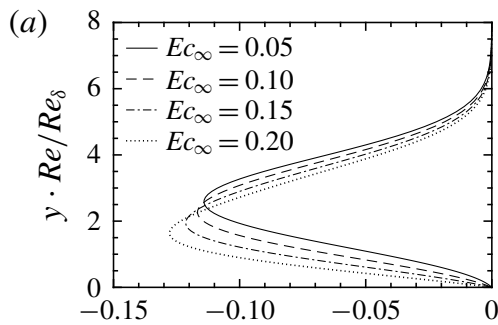

(b)
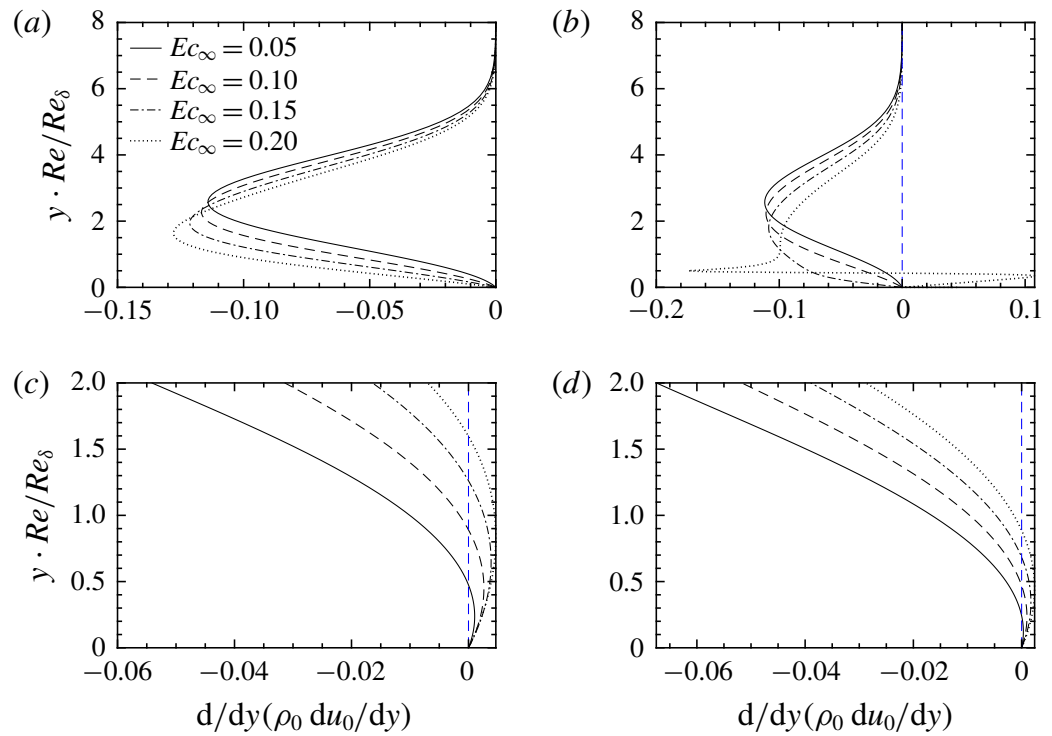

FIgURE 18. (Colour online) Generalized derivatives of the base flow $\mathrm{d}\left(\rho_{0} \mathrm{~d} u_{0} / \mathrm{d} y\right) / \mathrm{d} y$ for cases (a) T260, (b) T280, (c) T320 and (d) T340.

$$
\left.\begin{array}{c}
\theta=\frac{2 M_{r} D M_{r} D u_{0}}{\left(1-M_{r}^{2}\right)^{2}\left(u_{0}-c\right)}+\frac{D\left(\rho_{0} D u_{0}\right)}{\rho_{0}\left(1-M_{r}^{2}\right)\left(u_{0}-c\right)}, \\
\xi=-\frac{2 M_{r} D M_{r}}{\left(1-M_{r}^{2}\right)^{2}}-\frac{D \rho_{0}}{\rho_{0}\left(1-M_{r}^{2}\right)}, \\
\eta=-\frac{1}{1-M_{r}^{2}} .
\end{array}\right\}
$$

It can be recognized that at the critical layer $y=y_{c}$, where $u_{0}=c$ and $M_{r}=0$, the term $D\left(\rho_{0} D u_{0}\right)$ must vanish such that $y=y_{c}$ is a regular singular point of the $\hat{v}$ equation (4.8). Therefore, $D\left(\rho_{0} D u_{0}\right)$ plays the same role as $D\left(D u_{0} / T_{0}\right)$ given by Lees \& Lin (1946) and provides the generalized inflection point criterion for non-ideal gases.

In figure 18 , we show the generalized derivatives $\mathrm{d}\left(\rho_{0} \mathrm{~d} u_{0} / \mathrm{d} y\right) / \mathrm{d} y$ for cases $\mathrm{T} 260$, T280, T320 and T340, each with four Eckert numbers. It indicates that like ideal gases, an inviscid mechanism exists in the supercritical regime, while the stability is viscous in nature in the subcritical regime. It is worth noting that a generalized inflection point suddenly appears once the base flow turns from the sub- to the transcritical regime. It suggests the inviscid nature of Mode II. Note in figure 16, if compared to Mode I, Mode II has the peak of fluctuations $(|\hat{\rho}|,|\hat{u}|,|\hat{p}|)$ around the generalized inflection point, suggesting its inviscid nature (Lees \& Lin 1946). In figure 19 we compare the generalized derivative of the transcritical base flow for pressures $p_{0}^{*}=78,80$ and 82 bar. It indicates that the closer to the critical point $\left(p_{c}^{*}=73.9\right.$ bar), the more inflectional the base flow becomes, and the more unstable the flow in the transcritical regime.

\subsection{Oblique perturbations}

The LST and DNS studies in previous sections have both focused on the 2-D perturbations where $\beta=0$. Here we comment on the oblique perturbations. Examples 
(a)

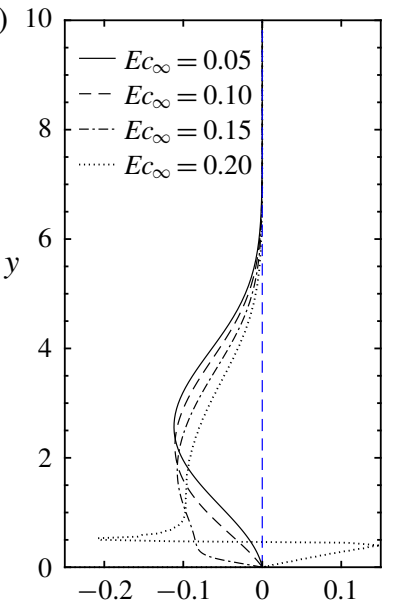

(b)

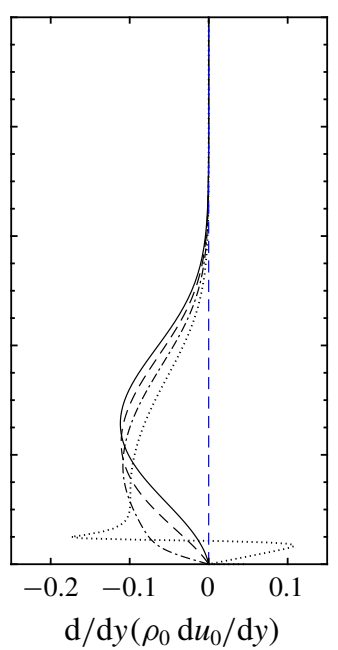

(c)

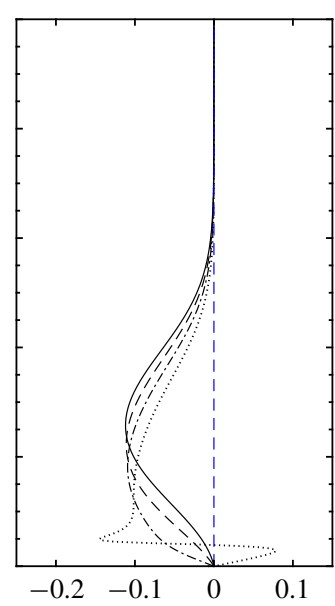

FIGURE 19. (Colour online) Generalized derivative of the laminar base flow with $T_{\infty}^{*}=$ $280 \mathrm{~K},(a) p_{0}^{*}=78$ bar, (b) $p_{0}^{*}=80$ bar and (c) $p_{0}^{*}=82$ bar.

(a)

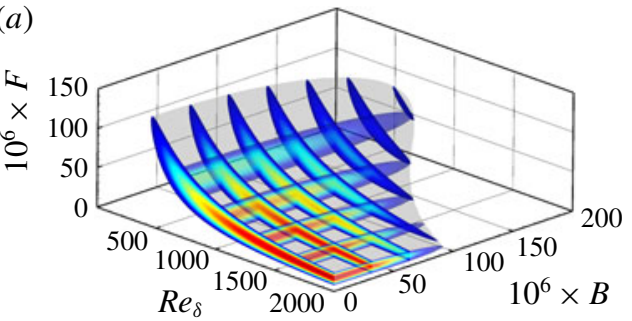

$-10^{3} \alpha_{i}$ (b)

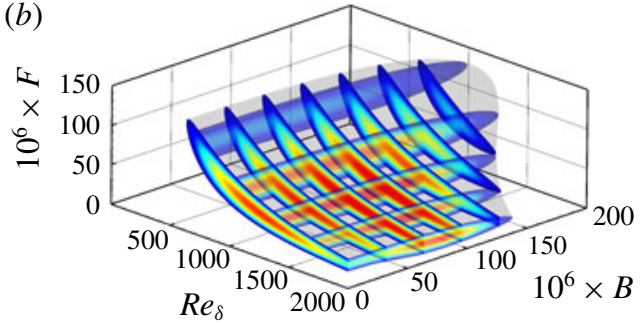

$-10^{3} \alpha_{i}$

$\begin{array}{lllllll}0 & 1 & 2 & 3 & 4 & 5 & 6\end{array}$

$\begin{array}{lllllllll}0 & 0.3 & 0.6 & 0.9 & 1.2 & 1.5 & 1.8\end{array}$

(c)

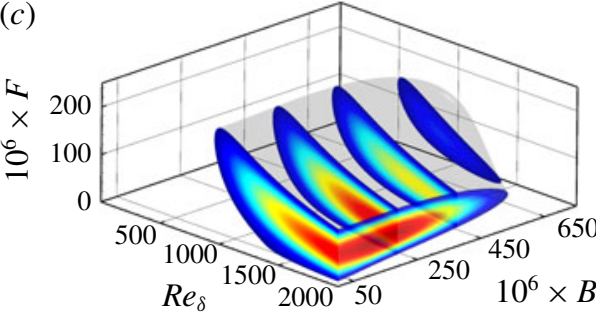

$-10^{3} \alpha_{i}$

$\begin{array}{lllllllllllll}0 & 1 & 2 & 3 & 4 & 5 & 6 & 7 & 8 & 9 & 10 & 11\end{array}$

FIgURE 20. (Colour online) Neutral surface along with slices of growth rate contours. $T_{\infty}^{*}=280 \mathrm{~K},(a) E c_{\infty}=0.05 ;$ (b) $E c_{\infty}=0.20$, Mode I; (c) $E c_{\infty}=0.20$, Mode II.

are provided in figure 20, which shows the neutral surface as well as slices of growth rate contours in the $\operatorname{Re}_{\delta}-F-B$ diagram; $B=\beta / \operatorname{Re}_{\delta}$ is defined as a global spanwise wavenumber. Figure $20(a)$ shows that with $T_{\infty}^{*}=280 \mathrm{~K}$, the single mode at $E c_{\infty}=0.05$ has a larger growth rate at $B=0$. Increase in $B$ results in a diminished 


$\begin{array}{lccccc}\text { Case } & \text { Growth rate ratio } & \beta_{m} & \text { Case } & \text { Growth rate ratio } & \beta_{m} \\ \text { T220E1 } & 1 & 0 & \text { T320E1 } & 1.0465 & 0.054 \\ \text { T220E2 } & 1 & 0 & \text { T320E2 } & 2.0501 & 0.078 \\ \text { T220E3 } & 1 & 0 & \text { T320E3 } & 10.5133 & 0.084 \\ \text { T220E4 } & 1 & 0 & \text { T320E4 } & 142.0449 & 0.084 \\ \text { T240E1 } & 1 & 0 & \text { T340E1 } & 1 & 0 \\ \text { T240E2 } & 1 & 0 & \text { T340E2 } & 1.0621 & 0.060 \\ \text { T240E3 } & 1 & 0 & \text { T340E3 } & 1.3769 & 0.072 \\ \text { T240E4 } & 1 & 0 & \text { T340E4 } & 2.2719 & 0.078 \\ \text { T260E1 } & 1 & 0 & \text { T360E1 } & 1 & 0 \\ \text { T260E2 } & 1 & 0 & \text { T360E2 } & 1.0090 & 0.036 \\ \text { T260E3 } & 1 & 0 & \text { T360E3 } & 1.1414 & 0.066 \\ \text { T260E4 } & 1 & 0 & \text { T360E4 } & 1.4162 & 0.072 \\ \text { T280E1 } & 1 & 0 & \text { T800E1 } & 1 & 0 \\ \text { T280E2 } & - & 0 & \text { T800E2 } & 1 & 0 \\ \text { T280E3 } & 1 & - & \text { T800E3 } & 1.0101 & 0.042 \\ \text { T280E4 (Mode I) } & 1.7016 & 0.150 & \text { T800E4 } & 1.0987 & 0.060 \\ \text { T280E4 (Mode II) } & 1 & 0 & & & \end{array}$

TABLE 2. Summary of the 3-D perturbation effects at $R e_{\delta}=2000 ; \beta_{m}$ indicates the optimal spanwise wavenumber at which the growth rate reaches maximum. Growth rate ratio gives the ratio of maximum growth rate at $\beta_{m}$ to the maximum value of $2-\mathrm{D}$ perturbations.

overall growth rate and a size-reduced neutral curve. This is however not true for Mode I at $E c_{\infty}=0.20$. It is shown in figure $20(b)$ that the largest growth happens at non-zero spanwise wavenumber $B$. Another example we show is Mode II in figure $20(c)$. This mode reaches its maximum growth rate at $B=0$.

A summary of the oblique effects is tabulated in table 2, where we show the results at $R e_{\delta}=2000$. The optimal spanwise wavenumber at which the growth rate reaches its maximum (over all frequencies) is termed $\beta_{m}$. The growth rate ratio shows the ratio between the maximum growth rate at $\beta=\beta_{m}$ and the value with $\beta=0$ (over all frequencies). Table 2 clearly indicates that 2 -D perturbations are the most unstable in the subcritical regime and in the transcritical regime for Mode II. In the supercritical regime for large $E c_{\infty}$ and in the transcritical regime for Mode I, oblique perturbations become more unstable.

In terms of eigenfunctions, figure 21 compares profiles of the oblique waves with their 2-D counterpart. Following table 2, we show case T280E4 (Mode I) and T320E3. Both reach maximum growth rate at a non-zero spanwise wavenumber. With $\beta=\beta_{m}$, the growth rate is considerably increased, and eigenfunctions are more mature and confined in the boundary layer. When the perturbation is oblique, the spanwise velocity perturbation $|\hat{w}|$ is present, while the density component $|\hat{\rho}|$ becomes relatively smaller. Note that the density profile has been scaled by a factor of 4 in panel (a) for a better display of the results.

\section{Conclusion}

Instabilities of compressible boundary-layer flows with non-ideal fluids are studied. As a typical example of a fluid in its highly non-ideal thermo-physical region, we 

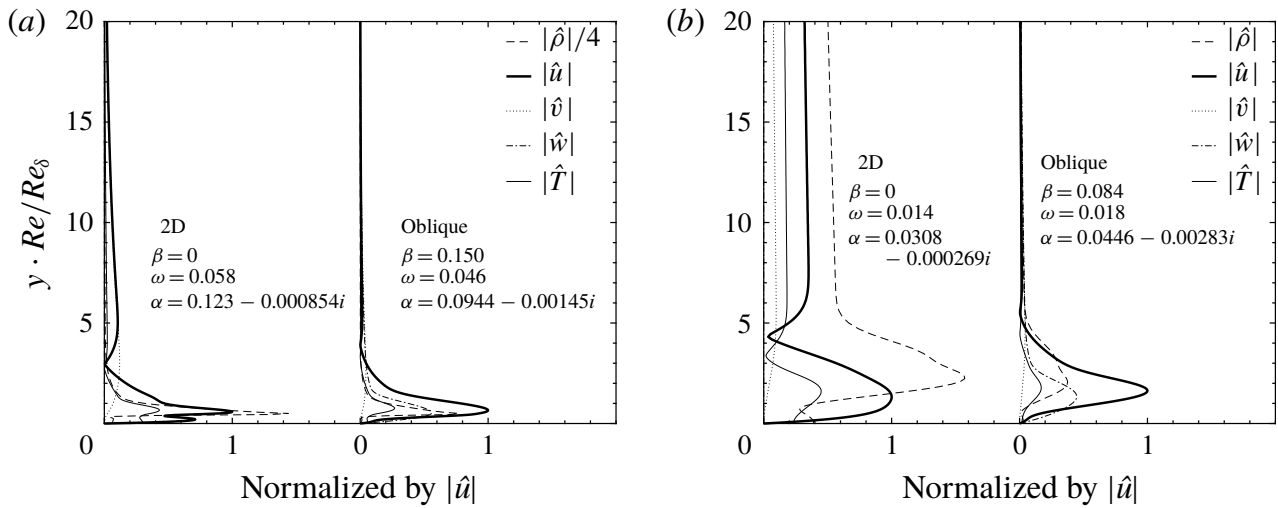

FIGURE 21. Comparison of the 2-D $(\beta=0)$ and oblique $\left(\beta=\beta_{m}\right)$ eigenfunctions at $R e_{\delta}=$ 2000. (a) Mode I in the transcritical regime, $T_{\infty}^{*}=280 \mathrm{~K}, E c_{\infty}=0.20 ;(b)$ the supercritical regime, $T_{\infty}^{*}=320 \mathrm{~K}, E c_{\infty}=0.15$. $\omega$ is chosen such that each mode reaches its maximum growth rate.

consider carbon dioxide $\left(\mathrm{CO}_{2}\right)$ at a supercritical pressure of 80 bar. The investigation is accomplished using linear stability theory (LST), direct numerical simulation (DNS) and the inviscid analysis, which are based on the compressible Navier-Stokes equations expressed for a generic fluid. To account for non-ideal-gas effects, the thermodynamic and transport properties as well as the equation of state are obtained from the NIST database and tabulated for computational efficiency. In terms of the Widom line $\left(T_{p c}^{*}=307.7 \mathrm{~K}\right.$ at $\left.80 \mathrm{bar}\right)$, the study has covered the sub-, trans- and supercritical temperature regimes, which are determined by the specified free-stream temperature $\left(T_{\infty}^{*}=220,240,260,280,320,340,360\right.$ and $\left.800 \mathrm{~K}\right)$, adiabatic wall boundary conditions and the Eckert number $E c_{\infty}$ that controls the viscous heating. The laminar base flows are given by the self-similar solutions of the boundary-layer equation and validated by DNS.

Besides stabilization of compressibility effects (increase $E c_{\infty}$ ), the boundary-layer flow is further stabilized by non-ideal-gas effects in the subcritical or supercritical regime. In either regime, the temperature profile remains below or above $T_{p c}^{*}$, the stabilization is more prominent when $T_{\infty}^{*}$ is closer to $T_{p c}^{*}$ and/or $E c_{\infty}$ is increased. However, the flow is considerably destabilized in the transcritical regime through a new unstable mode (Mode II) found in addition to the conventional mode (Mode I). In this regime, the dual modes co-exist, and their neutral curves partly overlap with each other. The growth rate of Mode II increases vigorously with $E c_{\infty}$ and can become an order of magnitude larger than Mode I. The unstable band of frequency and Reynolds number $R e_{\delta}$ for Mode II are much larger. DNS has witnessed quantitatively agreements with LST and the co-existence of the dual modes.

A summary of result is given in figure 22. For each case, the temperature ranges of the base flows (from free stream to the wall) are denoted with line segments. In all three regimes, density perturbation becomes the dominating components (absolute value of the perturbations) when compressibility and non-ideal-gas effects are strong (when $T_{\infty}^{*}$ is closer to $T_{p c}^{*}$ and $E c_{\infty}$ is large). The reason lies in massive density gradient $\partial \rho_{0} / \partial y$ of the laminar flow and the balancing mechanism of the linearized continuity equation. The other cases are dominated by the streamwise velocity component. The two arrows indicate the stabilization in the sub- and supercritical 


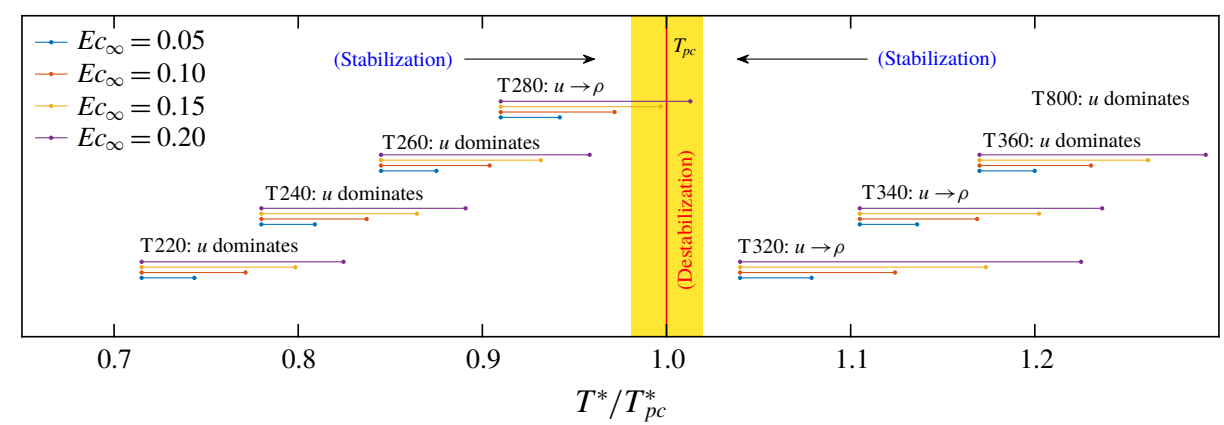

FIGURE 22. (Colour online) Summary of the non-ideal-gas effects on boundary-layer instability. Horizontal line segments stand for the temperature range of the base flow for each case. The red vertical line shows the pseudo-critical temperature $T_{p c}^{*}=307.7 \mathrm{~K}$.

regimes. Note that the current study is up to $E c_{\infty}=0.20(M a \leqslant 2)$ and therefore not influenced by hypersonic effects. In hypersonic flows, temperature perturbation can become the most prominent for ideal-gas flows (for example Görtler instability at $M a=6$ reported in Ren \& Fu 2015).

The oblique perturbations are also commented on in this study. In the subcritical regime, 2-D perturbations are more unstable. While in the supercritical regime, oblique perturbations are more important unless the free-stream temperature is large or/and the Eckert is small so that the flow is close to isothermal. In the transcritical regime, Mode I and Mode II are more important in three and two dimensions, respectively.

The inviscid analysis shows that in the transcritical regime, Mode II is not caused by the trapped acoustic waves which is deemed to give rise to higher modes in hypersonic flows. We show that the generalized inflection point criterion expressed in density $D\left(\rho_{0} D U_{0}\right)$ is valid for non-ideal gases. As result, an inviscid mechanism is present in the trans- and supercritical regimes in contrast to the subcritical regime which contains the viscous instability only.

\section{Acknowledgements}

J.R. and R.P. thank the Netherlands Organization for Scientific Research (NWO) for funding this research through the grant with project number 14711 and for the access to large-scale computing facilities through the grant with the dossier number SSH-22313.

\section{Supplementary movie}

Supplementary movie is available at https://doi.org/10.1017/jfm.2019.348.

\section{Appendix A. Fluid property tables}

This study employs the look-up table method to obtain the fluid properties needed for the laminar base flow, linear stability analysis as well as the direct numerical simulation. The NIST REFPROP library (Lemmon, Huber \& McLinden 2013; Span \& Wagner 2003) is adopted to generate the tables. The one-dimensional table is obtained by keeping pressure constant and is used for the self-similar solution and linear stability analysis. Figure 23 shows part of the table $\left(\rho^{*}, \operatorname{Pr}, \mu^{*}\right.$ and $\left.\kappa^{*}\right)$. 


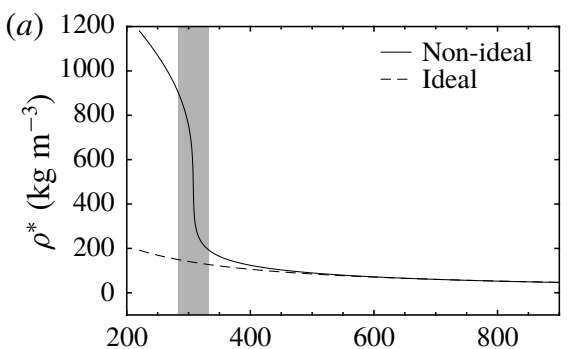

(b)
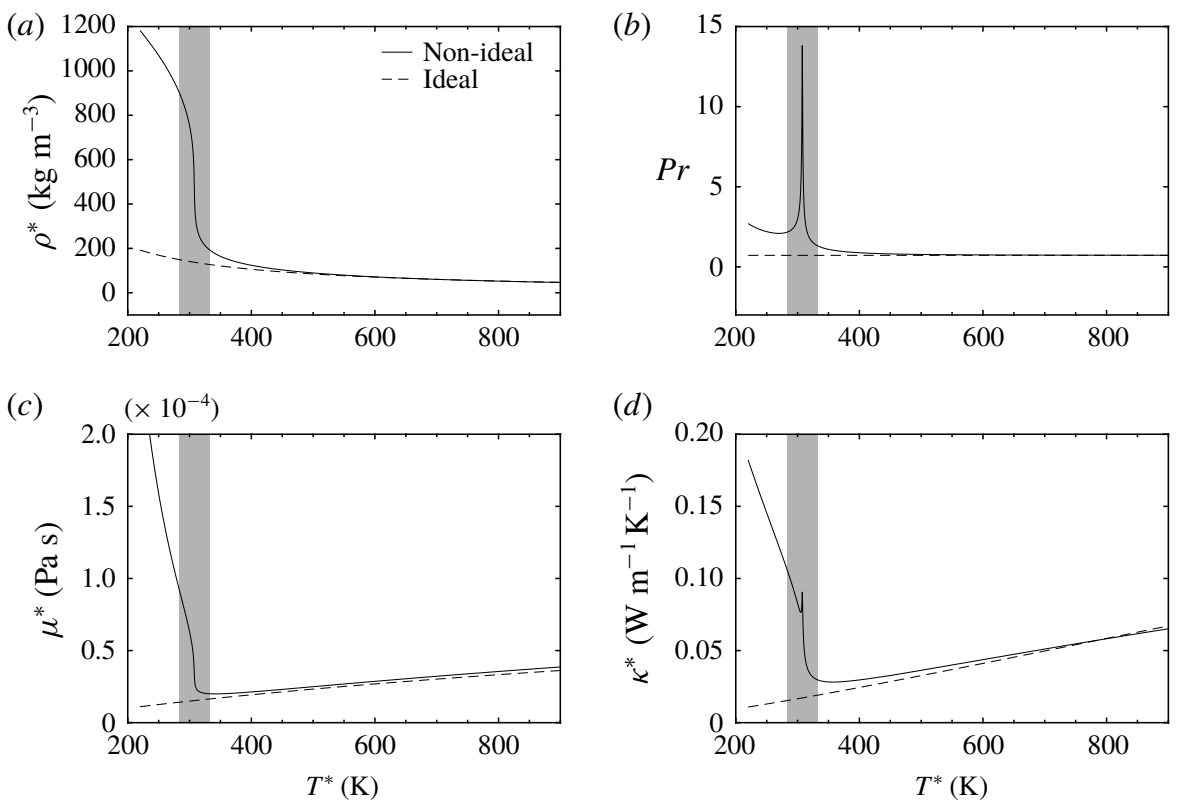

FIgURE 23. Visualization of the one-dimensional property table for $\mathrm{CO}_{2}$ at $p^{*}=80$ bar. Panels show density $(a)$, Prandtl number $(b)$, viscosity $(c)$ and thermal conductivity $(d)$ as functions of temperature. The shaded area indicates the region close to the pseudo-critical point where non-ideal-gas effects are prominent.

Dashed lines show the value under the ideal-gas assumption. It is obvious that the non-ideal-gas effects are most prominent near the critical point, and the fluid approaches the ideal gas regime only when the temperature is sufficiently large. The derivatives of the properties, such as $\partial \mu / \partial \rho, \partial^{2} p / \partial T^{2}$ are also important in building the stability operator. The gradients are determined using a second-order finite differences method (numerical details can be found in Ren et al. 2019).

In DNS, two-dimensional tables are required to obtain the properties. The 2-D tables are generated in the $\rho^{*}-e^{*}$ diagram. Figure 24 shows the 2-D table for pressure, temperature, viscosity and thermal conductivity as functions of $\rho^{*}$ and $e^{*}$. Note that in the DNS for different free-stream temperatures, the range of $\rho^{*}$ and $e^{*}$ have been adjusted so that the table maintains high accuracy and proper size. During the integration of the $\mathrm{N}-\mathrm{S}$ equations, only the data very close to the isobar are actually used. The data in the two-phase region are not determinant and have been assigned a value of -1 for all the properties. It has been checked that the data in the two-phase region are not used.

\section{Appendix B. Validation of the self-similar solution}

The self-similar solution to the boundary-layer equation with highly non-ideal gas is validated by comparing with the DNS. Figure 25 shows an example in the transcritical regime $\left(T_{\infty}^{*}=280 \mathrm{~K}, E c_{\infty}=0.20\right)$, of which non-ideal-gas effects are the most intense. The DNS is performed by integrating the $\mathrm{N}-\mathrm{S}$ equations from the self-similar solution as initial fields until the flow is steady and fully developed. Figure 25 shows a perfect match with DNS which justifies the self-similar solution. 

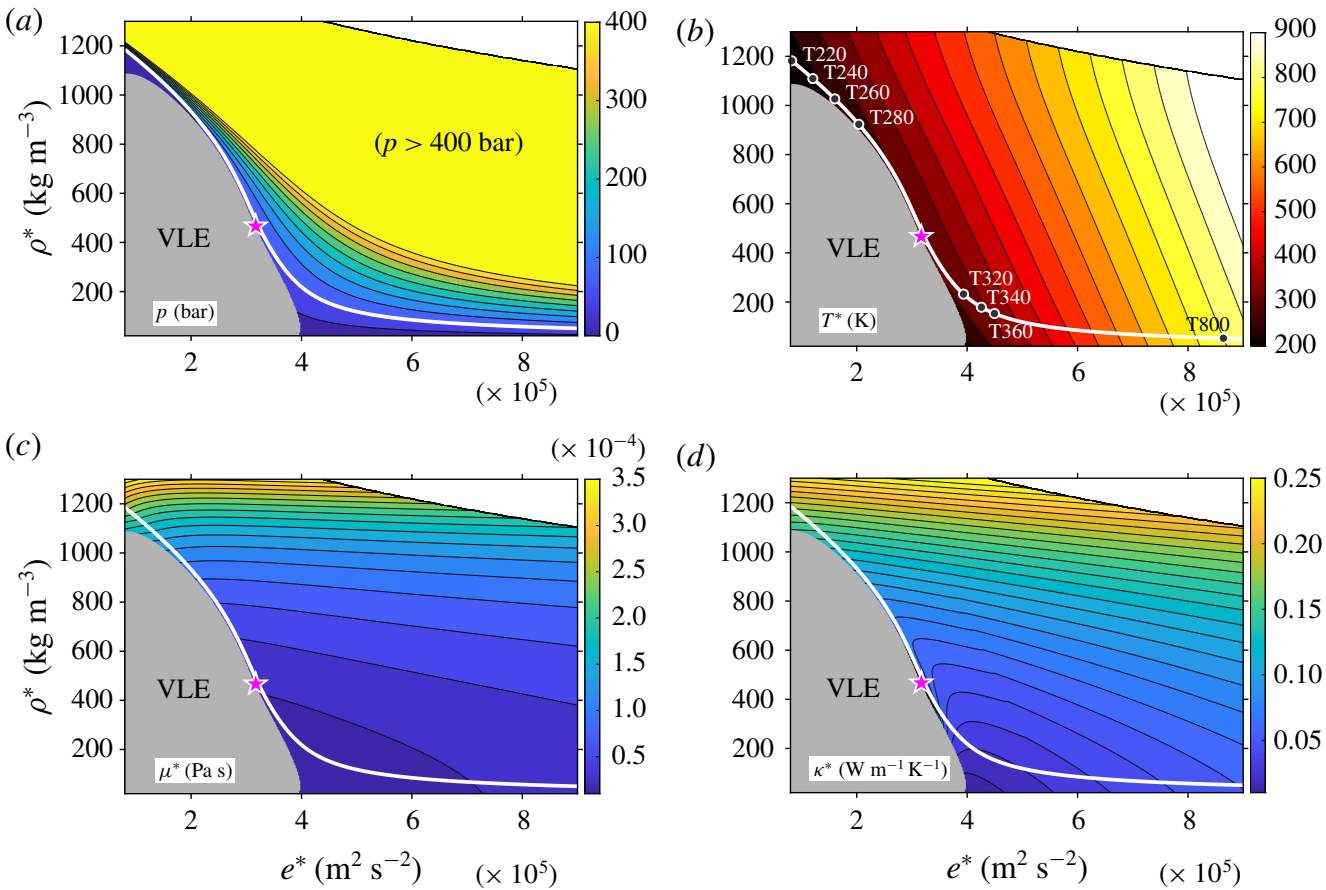

FIgURE 24. (Colour online) Visualization of the two-dimensional property table for $\mathrm{CO}_{2}$. Panels show pressure $(a)$, temperature $(b)$, viscosity $(c)$ and thermal conductivity $(d)$ as functions of internal energy $e^{*}$ and density $\rho^{*}$. The white line in each panel shows the isobar of 80 . The star indicates the critical point. VLE stands for the region of vapourliquid equilibrium.
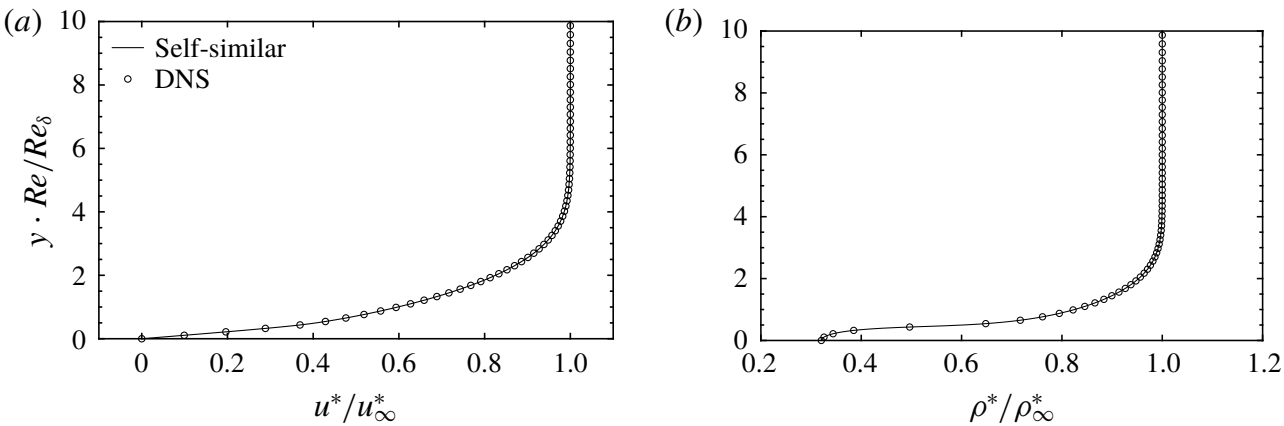

FIGURE 25. Validation of the self-similar solution by DNS. The flow parameters are $T_{\infty}^{*}=$ $280 \mathrm{~K}, E c_{\infty}=0.20$.

\section{Appendix C. DNS validation in the subcritical regime}

The results reported in this appendix serve as DNS validations of the LST prediction in the subcritical regime (T240 and T280), except for the case with $T_{\infty}^{*}=280 \mathrm{~K}$ and $E c_{\infty}=0.18$, which has just entered the transcritical regime. Figure 26(a1,b1) shows the neutral curves in $F-x$ diagram. The frequency $F=31 \times 10^{-6} / F=15 \times$ $10^{-6}$ cuts through the corresponding neutral curves and is introduced into the laminar 


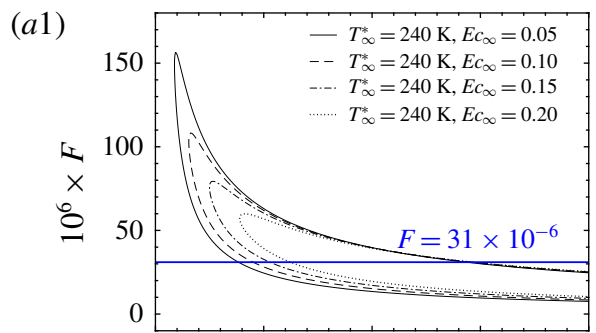

(b1)
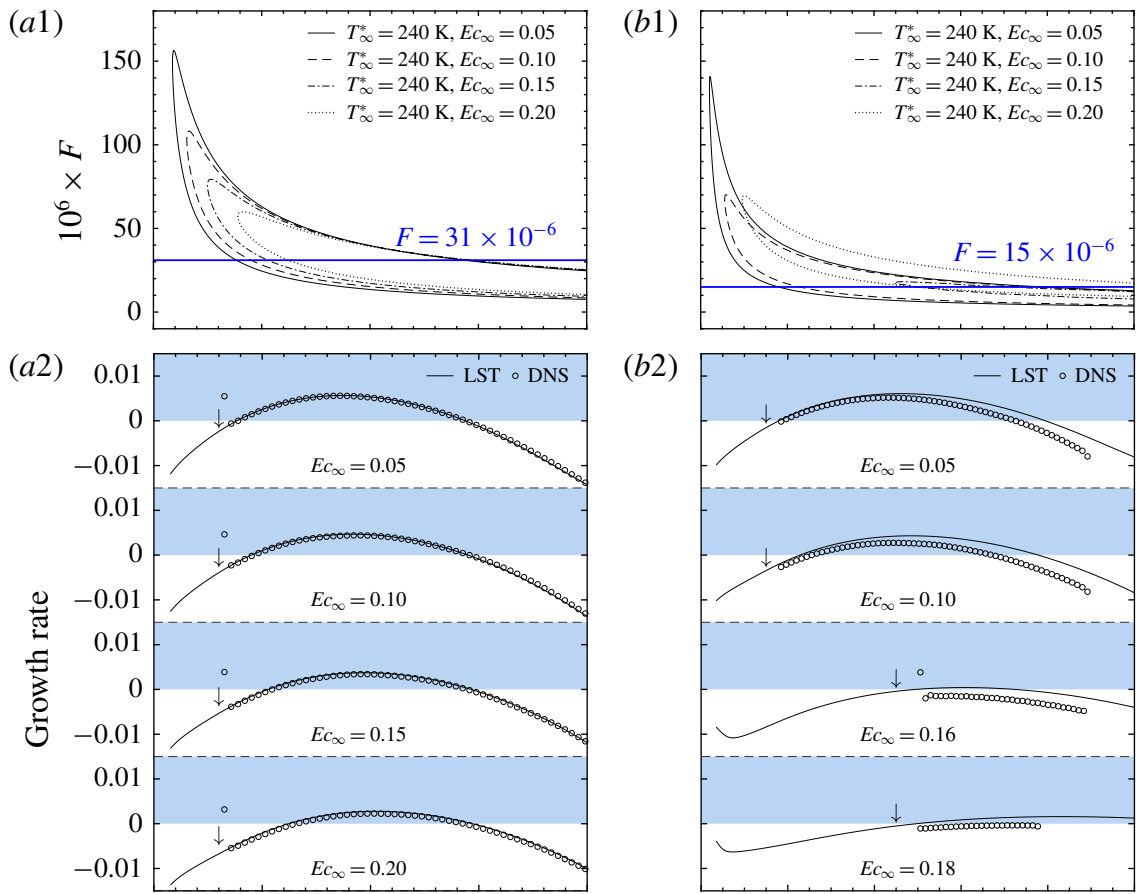

(b2)

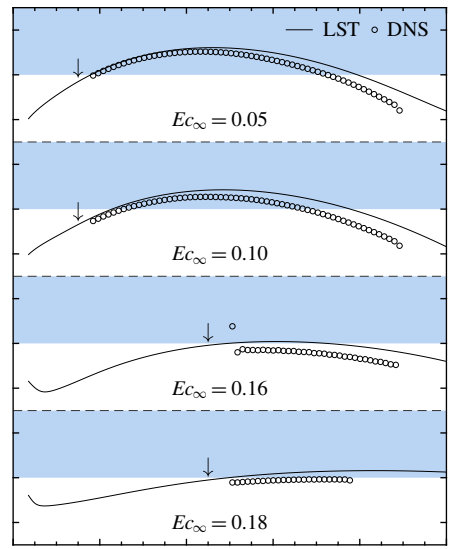

(a3)

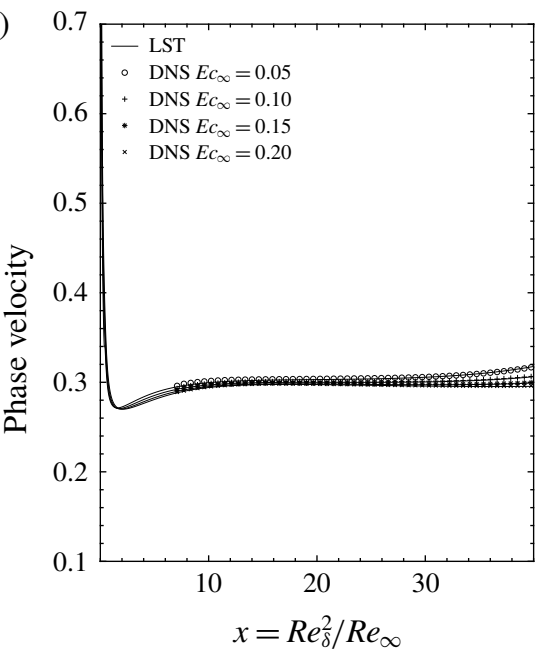

(b3)

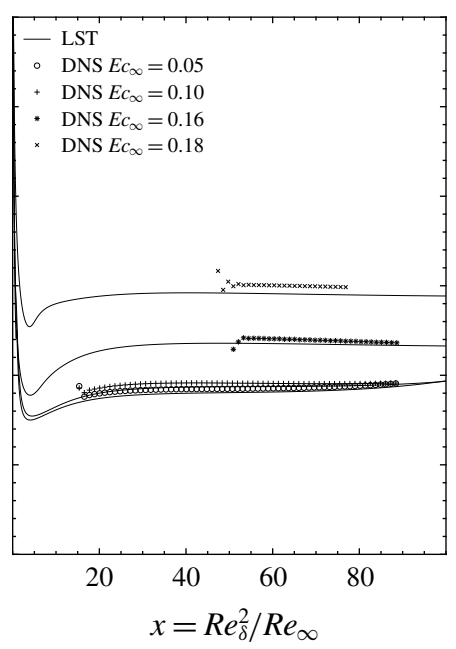

FIGURE 26. (Colour online) DNS validation in the subcritical regime with $T_{\infty}^{*}=240 \mathrm{~K}$ $(a 1-a 3)$ and $T_{\infty}^{*}=280 \mathrm{~K}(b 1-b 3)$. Panels $(a 1, b 1)$ show the neutral curve in the $F-x$ diagram. The blue solid line indicates the frequency of wall blowing/suction introduced to excite the T-S wave. Panels $(a 2, b 2, a 3, b 3)$ provide comparisons of the growth rate and phase velocity between DNS and LST. The arrows in $(a 2, b 2)$ indicate the position where wall blowing/suction is introduced.

flow via wall blowing/suction at the streamwise location indicated with an arrow in figure 26(a2,b2). Since a larger $R e_{\delta}$ is needed for the $E c_{\infty}=0.16$ and 0.18 cases of T280, and to better resolve these two cases, a smaller domain is used. The results have been verified to be mesh independent for all cases. Comparing the results, DNS and 
LST give rather satisfactory matches, verifying the stabilization of the non-ideal-gas effects in the subcritical regime, as well as the destabilization of Mode I when the flow enters transcritical regime $\left(T_{\infty}^{*}=280 \mathrm{~K}, E c_{\infty}=0.18\right)$. It is worth noting, in the subcritical regime, when $T_{\infty}^{*} \ll T_{p c}^{*}$, the phase velocity becomes much less sensitive to $E c_{\infty}$.

\section{Appendix D. Mathematical proof of (4.7)}

To derive the relative Mach number, the following rules (see Thorade \& Saadat 2013) for the partial derivatives have been used,

$$
\left.\begin{array}{c}
\left(\left.\frac{\partial x}{\partial b}\right|_{y}\right)=\left(\left.\frac{\partial b}{\partial x}\right|_{y}\right)^{-1} \quad \text { (Reciprocity rule), } \\
\left(\left.\frac{\partial a}{\partial b}\right|_{x}\right)=\left(\left.\frac{\partial a}{\partial y}\right|_{x}\right)\left(\left.\frac{\partial b}{\partial y}\right|_{x}\right)^{-1} \quad \text { (Chain rule), } \\
\left(\left.\frac{\partial x}{\partial y}\right|_{c}\right)=-\left(\left.\frac{\partial c}{\partial y}\right|_{x}\right)\left(\left.\frac{\partial c}{\partial x}\right|_{y}\right)^{-1} \quad \text { (Triple product rule). }
\end{array}\right\}
$$

We omit the subscript ' 0 ' for base flow below. Using these rules, the speed of sound squared can be written as

$$
\begin{aligned}
\left(\left.\frac{\partial p}{\partial \rho}\right|_{s}\right) & =\left(\left.\frac{\partial p}{\partial \rho}\right|_{e}\right)-\left(\left.\frac{\partial p}{\partial e}\right|_{\rho}\right)\left(\left.\frac{\partial s}{\partial \rho}\right|_{e}\right)\left(\left.\frac{\partial s}{\partial e}\right|_{\rho}\right)^{-1} \\
& =\left(\left.\frac{\partial p}{\partial \rho}\right|_{e}\right)+\left(\left.\frac{\partial p}{\partial e}\right|_{\rho}\right)\left(\left.\frac{\partial e}{\partial \rho}\right|_{s}\right) \\
& =\left(\left.\frac{\partial p}{\partial \rho}\right|_{e}\right)-\left(\left.\frac{\partial e}{\partial \rho}\right|_{s}\right)\left(\left.\frac{\partial \rho}{\partial e}\right|_{p}\right)\left(\left.\frac{\partial p}{\partial \rho}\right|_{e}\right) \\
& =\left(\left.\frac{\partial p}{\partial \rho}\right|_{e}\right)-\left(\left.\frac{\partial e}{\partial \vartheta}\right|_{s}\right)\left(\left.\frac{\partial \vartheta}{\partial e}\right|_{p}\right)\left(\left.\frac{\partial p}{\partial \rho}\right|_{e}\right) \\
& =\left(\left.\frac{\partial \rho}{\partial p}\right|_{e}\right)^{-1}\left[1-\left(\frac{\partial e}{\partial \vartheta} \mid\right)\left(\left.\frac{\partial \vartheta}{\partial e}\right|_{p}\right)\right]_{s} \\
& =\left(\left.\frac{\partial \rho}{\partial p}\right|_{e}\right)^{-1}\left[1-\frac{p}{\rho^{2}}\left(\left.\frac{\partial \rho}{\partial e}\right|_{p}\right)\right]
\end{aligned}
$$

Note that according to the first law of thermodynamics, $p=-\left(\partial e /\left.\partial \vartheta\right|_{s}\right)$. The relative Mach number in (4.7) can thus be derived from (4.6).

\section{REFERENCES}

Anderson, J. D. JR 2000 Hypersonic and High Temperature Gas Dynamics. AIAA.

Brown, B. P. \& Argrow, B. M. 2000 Application of Bethe-Zel'dovich-Thompson fluids in organic Rankine cycle engines. J. Propul. Power 16 (6), 1118-1124. 
Brunner, G. 2010 Applications of supercritical fluids. Annu. Rev. Chem. Biomol. Engng 1, 321-342.

Chang, C.-L., Vinh, H., Malik, M., Malik, M., Chang, C.-L. \& Vinh, H. 1997 Hypersonic boundary-layer stability with chemical reactions using PSE. In 28th Fluid Dynamics Conference, p. 2012. American Institute of Aeronautics and Astronautics.

Facchini, G., Favier, B., Le Gal, P., Wang, M. \& Le Bars, M. 2018 The linear instability of the stratified plane Couette flow. J. Fluid Mech. 853, 205-234.

Fedorov, A. 2011 Transition and stability of high-speed boundary layers. Annu. Rev. Fluid Mech. 43 (1), 79-95.

Fedorov, A. \& Tumin, A. 2011 High-speed boundary-layer instability: old terminology and a new framework. AIAA J. 49 (8), 1647-1657.

Franko, K., MacCormack, R. \& Lele, S. 2010 Effects of chemistry modeling on hypersonic boundary layer linear stability prediction. In 40th Fluid Dynamics Conference and Exhibit, p. 4601. American Institute of Aeronautics and Astronautics.

Govindarajan, R. \& SAHU, K. C. 2014 Instabilities in viscosity-stratified flow. Annu. Rev. Fluid Mech. 46, 331-353.

Huang, P. G., Coleman, G. N. \& Bradshaw, P. 1995 Compressible turbulent channel flows: DNS results and modelling. J. Fluid Mech. 305, 185-218.

Hudson, M. L., ChokAni, N. \& CANDler, G. V. 1997 Linear stability of hypersonic flow in thermochemical nonequilibrium. AIAA J. 35 (6), 958-964.

JOHnSON, H. \& CANDLER, G. 1999 PSE analysis of reacting hypersonic boundary layer transition. In 30th Fluid Dynamics Conference, p. 3793. American Institute of Aeronautics and Astronautics.

KAWAI, S. 2016 Direct numerical simulation of transcritical turbulent boundary layers at supercritical pressures with strong real fluid effects. In 54th AIAA Aerospace Sciences Meeting, p. 1934. American Institute of Aeronautics and Astronautics.

LEES, L. \& LIN, C. C. 1946 Investigation of the stability of the laminar boundary layer in a compressible fluid. NACA Tech. Note 1115.

Lemmon, E. W., Huber, M. L. \& McLinden, M. O. 2013 NIST Standard Reference Database 23: Reference Fluid Thermodynamic and Transport Properties REFPROP, Version 9.1. National Institute of Standards and Technology. Available at: http://www.nist.gov/srd/nist23.cfm.

Lyttle, I. \& ReEd, H. 2005 Sensitivity of second-mode linear stability to constitutive models within hypersonic flow. In 43rd AIAA Aerospace Sciences Meeting and Exhibit, p. 889. American Institute of Aeronautics and Astronautics.

MACK, L. M. 1984 Boundary-layer linear stability theory. In AGARD Report No. 709: Special Course on Stability and Transition of Laminar Flow. AGARD.

MALIK, M. R. 2003 Hypersonic flight transition data analysis using parabolized stability equations with chemistry effects. J. Spacecr. Rockets 40 (3), 332-344.

Matik, M. R. \& Anderson, E. C. 1991 Real gas effects on hypersonic boundary-layer stability. Phys. Fluids A 3 (5), 803-821.

MARXen, O., IACCARINo, G. \& MAgin, T. E. 2014 Direct numerical simulations of hypersonic boundary-layer transition with finite-rate chemistry. J. Fluid Mech. 755, 35-49.

Marden, O., Magin, T., IAccarino, G. \& Shaqfeh, E. S. G. 2011 A high-order numerical method to study hypersonic boundary-layer instability including high-temperature gas effects. Phys. Fluids 23 (8), 084108.

Marden, O., Magin, T. E., Shaqfeh, E. S. G. \& Iaccarino, G. 2013 A method for the direct numerical simulation of hypersonic boundary-layer instability with finite-rate chemistry. J. Comput. Phys. 255, 572-589.

Mortensen, C. H. \& ZHONG, X. 2016 Real-gas and surface-ablation effects on hypersonic boundarylayer instability over a blunt cone. AIAA J. 54 (3), 980-998.

Nagarajan, S., Lele, S. K. \& Ferziger, J. H. 2003 A robust high-order compact method for large eddy simulation. J. Comput. Phys. 191 (2), 392-419.

Pecnik, R. \& Patel, A. 2017 Scaling and modelling of turbulence in variable property channel flows. J. Fluid Mech. 823, R1.

PizZARELli, M. 2018 The status of the research on the heat transfer deterioration in supercritical fluids: a review. Intl Commun. Heat Mass Transfer 95, 132-138. 
Raju, M., Banuti, D. T., Ma, P. C. \& Ihme, M. 2017 Widom lines in binary mixtures of supercritical fluids. Sci. Rep. 7 (1), 3027.

REN, J. \& FU, S. 2015 Secondary instabilities of Görtler vortices in high-speed boundary layer flows. J. Fluid Mech. 781, 388-421.

Ren, J., FU, S. \& HANIFI, A. 2016 Stabilization of the hypersonic boundary layer by finite-amplitude streaks. Phys. Fluids 28 (2), 024110.

REN, J., FU, S. \& PECNIK, R. 2019 Linear instability of Poiseuille flows with highly non-ideal fluids. J. Fluid Mech. 859, 89-125.

Sameen, A. \& Govindarajan, R. 2007 The effect of wall heating on instability of channel flow. J. Fluid Mech. 577, 417-442.

SChlichting, H. \& Gersten, K. 2017 Boundary-Layer Theory. Springer.

Schmid, P. J. \& Henningson, D. S. 2001 Stability and Transition in Shear Flows. Springer.

Sciortino, F., Poole, P. H., Essmann, U. \& Stanley, H. E. 1997 Line of compressibility maxima in the phase diagram of supercooled water. Phys. Rev. E 55 (1), 727.

Span, R. \& Wagner, W. 2003 Equations of state for technical applications. I. Simultaneously optimized functional forms for nonpolar and polar fluids. Intl J. Thermophys. 24, 1-39.

Stemmer, C., Birrer, M. \& Adams, N. A. 2017 Disturbance development in an obstacle wake in a reacting hypersonic boundary layer. J. Spacecr. Rockets 54 (4), 945-960.

StUCKeRT, G. \& ReED, H. L. 1994 Linear disturbances in hypersonic, chemically reacting shock layers. AIAA J. 32 (7), 1384-1393.

Thorade, M. \& SAAdat, A. 2013 Partial derivatives of thermodynamic state properties for dynamic simulation. Environ. Earth Sci. 70 (8), 3497-3503.

WANG, X. 2017 Non-equilibrium effects on the stability of a Mach 10 flat-plate boundary layer. In 8th AIAA Theoretical Fluid Mechanics Conference, p. 3162. American Institute of Aeronautics and Astronautics.

WANG, X. \& YANG, V. 2017 Supercritical mixing and combustion of liquid-oxygen/kerosene bi-swirl injectors. J. Propul. Power 33 (2), 316-322.

Zhong, X. \& WANG, X. 2012 Direct numerical simulation on the receptivity, instability, and transition of hypersonic boundary layers. Annu. Rev. Fluid Mech. 44 (1), 527-561. 